\title{
NUMERICAL STUDY FOR THE NUCLEATION OF ONE-DIMENSIONAL STOCHASTIC CAHN-HILLIARD DYNAMICS*
}

\author{
WEI ZHANG ${ }^{\dagger}$, TIEJUN LI ${ }^{\ddagger}$, AND PINGWEN ZHANG $\S$
}

\begin{abstract}
We consider the nucleation of one-dimensional stochastic Cahn-Hilliard dynamics with the standard double well potential. We design the string method for computing the most probable transition path in the zero temperature limit based on large deviation theory. We derive the nucleation rate formula for the stochastic Cahn-Hilliard dynamics through finite dimensional discretization. We also discuss the algorithmic issues for calculating the nucleation rate, especially the high dimensional sampling for computing the determinant ratios.
\end{abstract}

Key words. Cahn-Hilliard equation, large deviation theory, nucleation rate, string method, Metropolis-Hastings algorithm.

AMS subject classifications. 60H35, 65Z05, 82C26.

\section{Introduction}

Nucleation is a universal and important event during phase transitions. A system initially located at a metastable state will fluctuate around this metastable state under thermal noise perturbation. Usually this process occurs on an exponentially long time scale, and the phase transition occurs when the noise happens to be large enough to carry the system out of the basin of attraction of this metastable state. As a result the system transitions to another metastable state and similar situation continues. This typical behavior can be characterized as an energy barrier crossing event and the study of these rare events has attracted considerable attentions in physics, chemistry, and applied mathematics; see [1, 2, 3] and the references therein.

When studying nucleation events with smooth energy landscape in phase transition phenomena, the following three questions are typically relevant:

Q1. What is the most probable transition path and how does one compute it?

Q2. What is the transitional state (saddle point) along the path?

Q3. How large is the nucleation rate for the considered process?

These questions are of prime importance in understanding the transition events and lots of work has been done related to them. If we restrict ourselves to the overdamped Langevin dynamics, there have been some answers: For Q1, theoretically, the large deviation theory [4] in probability theory is a suitable framework for the study of the transition paths in the zero temperature limit, and can be achieved by minimizing an action functional $[5,6]$. Numerically, some efficient algorithms such as the string method [7] and the NEB method [8] have been developed to locate the most probable transition path. For Q2, the saddle point can be automatically identified from the

*Received: September 10, 2011; accepted (in revised form): February 3, 2012. Communicated by Eric Vanden-Eijnden.

${ }^{\dagger}$ Beijing International Center for Mathematical Research (BICMR), Laboratory of Mathematics and Applied Mathematics (LMAM) and School of Mathematical Sciences, Peking University, Beijing 100871, P.R. China (wzhangpku@gmail.com).

$\ddagger$ Beijing International Center for Mathematical Research (BICMR), Laboratory of Mathematics and Applied Mathematics (LMAM) and School of Mathematical Sciences, Peking University, Beijing 100871, P.R. China (tieli@pku.edu.cn).

$\S$ Beijing International Center for Mathematical Research (BICMR), Laboratory of Mathematics and Applied Mathematics (LMAM) and School of Mathematical Sciences, Peking University, Beijing 100871, P.R. China (pzhang@pku.edu.cn). 
obtained connecting path. But in the case that the saddle point is of independent interest, the dimer method and GAD method can be used $[9,10]$. For Q3, the rate can be obtained by studying the mean first exit time of the stochastic differential equations or given by Kramer's reaction rate theory [1, 11], or more sophisticatedly by the transition path theory developed recently in [3, 12].

While studying phase transition phenomena, the Allen-Cahn and Cahn-Hilliard equations are two typical models for describing the non-conservative and conservative order parameters, which are classified as Model A and Model B in [13], respectively. In this paper, we focus on the nucleation of the stochastic Cahn-Hilliard dynamics (Model B) and aim to develop general algorithms and computational strategies to answer the three key questions proposed above. The considered dynamics will be only one dimensional with Ginzburg-Landau type double well potential in the current paper and the applications to higher dimensions and more complex models will be studied in continued publications.

In the mathematics community, the analytical study of the stochastic CahnHilliard equation has been investigated by many authors [14, 15, 16, 17, 18]. Among these studies, the large deviation type estimates and the dynamical system analysis on the structure of the attractors are the most relevant to the study in this paper. By drawing a framework similar to those in $[5,7]$, we propose the string method to compute the most probable transition path for the stochastic Cahn-Hilliard dynamics with periodic or Neumann boundary conditions in the zero temperature limit. Based on the obtained transition path, the transition state can be located easily. We also derive the nucleation rate formula and design the related computational strategies.

The rest of this paper is organized as follows: in Section 2, we formulate the stochastic Cahn-Hilliard equation and discretize it into finite-dimensional space. In Section 3, we get the most probable transition path by minimizing the action functional based on the large deviation theory. Then we propose the string method to locate the transition path and the saddle points. The nucleation rate formula and the related computational strategies are discussed in Section 4. In Section 5, we present numerical results for the one dimensional Cahn-Hilliard equation. Further discussions and possible extensions are included in Section 6. Finally we make conclusions. All of the details for the derivation of the nucleation rate formula can be found in Appendix A.

\section{Problem set-up and preliminary results}

2.1. One-dimensional stochastic Cahn-Hilliard equation. We consider the 1D stochastic Cahn-Hilliard equation (or Cahn-Hilliard-Cook model) [14, 17]

$$
\frac{\partial u}{\partial t}=\frac{\partial^{2}}{\partial x^{2}} \frac{\delta F(u)}{\delta u}+\sqrt{2 \epsilon} \xi=\frac{\partial^{2}}{\partial x^{2}}\left(-\kappa^{2} \frac{\partial^{2} u}{\partial x^{2}}+u^{3}-u\right)+\sqrt{2 \epsilon} \xi,
$$

where $x \in(0,1)$ and $F(u)$ is the Ginzburg-Landau type energy functional

$$
F(u)=\int_{0}^{1}\left[\frac{\kappa^{2}}{2}\left(\frac{\partial u}{\partial x}\right)^{2}+f(u)\right] d x,
$$

which incorporates the contribution of both the interface and bulk energy through the gradient term and potential $f(u)$ respectively. The parameter $\kappa>0$ describes the interaction strength between two phases in alloy phase separation, which is in turn related to the thickness of the interface. The driving potential $f(u)$ is usually simplified to the well-known double well shape $f(u)=\left(u^{2}-1\right)^{2} / 4$ with two global 
minima at $u= \pm 1$. The noise term is $\xi=\partial_{x} \eta$, and $\eta(x, t)$ is the space-time Gaussian white noise satisfying

$$
E \eta(x, t)=0 \quad \text { and } \quad E \eta(x, t) \eta(y, s)=\delta(t-s) \delta(x-y) .
$$

The parameter $\epsilon>0$ describes the strength of the noise which is related to temperature.

The following two kinds of boundary conditions (BC) are typically considered in the literature (see Remark 2.1 for a discussion).

1. Neumann BC:

$$
\begin{aligned}
& \partial_{x} u=\partial_{x}^{3} u=0 \text { at } x=0,1, \\
& \eta(0, t)=\eta(1, t)=0 .
\end{aligned}
$$

2. Periodic BC:

$$
\begin{aligned}
& u, \partial_{x} u, \partial_{x}^{3} u \text { are periodic in } \mathbb{R} \text { with period } 1, \\
& \eta(x, t)=\eta(x+1, t) .
\end{aligned}
$$

For both boundary conditions, $\xi$ is a conservative noise in (2.1). To see this, we integrate equation $(2.1)$ over $[0,1]$ and use the boundary conditions, thus obtaining the following conservation relation:

$$
\int_{0}^{1} u(x, t) d x=\int_{0}^{1} u(x, 0) d x=m,
$$

for arbitrary $t>0$, as in the case of the deterministic Cahn-Hilliard equation. Defining

$$
\mathbb{E}=\left\{w(x) \mid w(x) \in L^{2}[0,1], \int_{0}^{1} w(x) d x=m\right\},
$$

we have $u(\cdot, t) \in \mathbb{E}, \forall t \geq 0$, and we define $\mathbb{P}=C_{\mathbb{E}}[0,+\infty)$ as the trajectory space of the continuous dynamics.

REMARK 2.1. Now we give a rationale for the proposed boundary conditions in this remark. Following [17], the Cahn-Hilliard equation can be obtained by considering

$$
\frac{\partial u}{\partial t}+\nabla \cdot J(x, t, u(t))=0
$$

where the flux $J$ is modeled by the Fick's law

$$
J=-\mu \nabla p
$$

and the chemical potential $p=\delta F(u) / \delta u$. The stochastic effect is taken into account by adding a random fluctuation to the flux:

$$
J=-\mu \nabla p+\vec{\eta}(x, t),
$$

where $\vec{\eta}(x, t)=\left(\eta^{1}(x, t), \eta^{2}(x, t), \ldots, \eta^{d}(x, t)\right), d$ is the dimension, $\eta^{i}(x, t)$ are independent space-time Gaussian white noises. For Neumann BC, $J \cdot \vec{n} \equiv 0$ is assumed to hold on the boundary and thus both the deterministic part and the stochastic part of the flux in normal direction are proposed to be zero:

$$
\nabla p \cdot \vec{n}=\vec{\eta}(x, t) \cdot \vec{n}=0 .
$$

For periodic BC, similarly, both the deterministic part $\nabla p \cdot \vec{n}$ and the stochastic part $\vec{\eta}(x, t) \cdot \vec{n}$ of the flux are proposed to be periodic.

In $1 \mathrm{D}, \vec{\eta}(x, t)=\eta(x, t)$ is a space-time white noise satisfying (2.3) and thus we obtain the boundary conditions (2.4) and (2.5). 
2.2. Discretization in finite dimensions. As in [17], to get the large deviation theory for the infinite dimensional case, we first need to consider the finite dimensional discretization and then take the continuum limit. This strategy is unavoidable especially when we want to compute the nucleation rate. For simplicity we discretize the interval $[0,1]$ uniformly. Define $\Delta x=1 / n, x_{i}=(i-1 / 2) \Delta x, x_{i}^{\prime}=$ $i \Delta x, i=1,2, \cdots, n$. Let

$$
X_{t}=\left(X_{t}^{1}, X_{t}^{2}, \cdots, X_{t}^{n}\right) \in \mathbb{R}^{n},
$$

where $X_{t}^{i}$ corresponds to the approximation of $u\left(x_{i}, t\right)$, respectively. The dynamical system (2.1) in continuous space is discretized to a generalized gradient system

$$
d X_{t}=-A \nabla V\left(X_{t}\right) d t+\sqrt{\frac{2 \epsilon}{\Delta x}} \sigma d W_{t}
$$

where the matrices $A$ and $\sigma$ are the matrix forms of the operators $-\frac{\partial^{2}}{\partial x^{2}}$ and $\frac{\partial}{\partial x}$ after discretization, which will be given below for different boundary conditions. The potential function $V(w)=F_{n}(w) / \Delta x$, where $w=\left(w_{1}, w_{2}, \cdots, w_{n}\right)^{T} \in \mathbb{R}^{n}$, and $F_{n}(w)$ is the discretized version of the functional $F(u)$ :

$$
F_{n}(w)=\left\{\begin{array}{l}
\left\{\sum_{i=2}^{n}\left[\frac{\kappa^{2}}{2}\left(\frac{w_{i}-w_{i-1}}{\Delta x}\right)^{2}+f\left(w_{i}\right)\right]+f\left(w_{1}\right)\right\} \Delta x, \text { for Neumann BC, } \\
\left\{\sum_{i=2}^{n}\left[\frac{\kappa^{2}}{2}\left(\frac{w_{i}-w_{i-1}}{\Delta x}\right)^{2}+f\left(w_{i}\right)\right]+\frac{\kappa^{2}}{2}\left(\frac{w_{n}-w_{1}}{\Delta x}\right)^{2}+f\left(w_{1}\right)\right\} \Delta x, \text { for periodic BC. }
\end{array}\right.
$$

Under Neumann BC, $W_{t}$ is an $(n-1)$-dimensional Brownian motion obtained by discretizing the white noise $\eta$ at $x_{i}(i=1,2, \cdots, n)$ by using values on $x_{i}^{\prime}(i=$ $1,2, \cdots, n-1) . A \in M_{n \times n}$ and $\sigma \in M_{n \times(n-1)}$ take the following forms:

$$
A=\frac{1}{\Delta x^{2}}\left(\begin{array}{cccccc}
1 & -1 & 0 & \cdots & 0 & 0 \\
-1 & 2 & -1 & 0 & \cdots & 0 \\
0 & \ddots & \ddots & \ddots & \ddots & 0 \\
0 & \cdots & -1 & 2 & -1 & 0 \\
0 & 0 & \cdots & -1 & 2 & -1 \\
0 & 0 & \cdots & 0 & -1 & 1
\end{array}\right), \sigma=\frac{1}{\Delta x}\left(\begin{array}{ccccc}
1 & 0 & 0 & \cdots & 0 \\
-1 & 1 & 0 & \cdots & 0 \\
0 & \ddots & \ddots & \ddots & 0 \\
0 & \cdots & -1 & 1 & 0 \\
0 & 0 & \cdots & -1 & 1 \\
0 & 0 & \cdots & 0 & -1
\end{array}\right) \text {. }
$$

For periodic BC, $W_{t}$ is an $n$-dimensional Brownian motion obtained by discretizing the white noise $\eta$ at $x_{i}(i=1,2, \cdots, n)$ by using values on $x_{i}^{\prime}(i=1,2, \cdots, n)$. $A, \sigma \in M_{n \times n}$ satisfy

$$
A=\frac{1}{\Delta x^{2}}\left(\begin{array}{cccccc}
2 & -1 & 0 & \cdots & 0 & -1 \\
-1 & 2 & -1 & 0 & \cdots & 0 \\
0 & \ddots & \ddots & \ddots & \ddots & 0 \\
0 & \cdots & -1 & 2 & -1 & 0 \\
0 & 0 & \cdots & -1 & 2 & -1 \\
-1 & 0 & \cdots & 0 & -1 & 2
\end{array}\right), \sigma=\frac{1}{\Delta x}\left(\begin{array}{cccccc}
1 & 0 & 0 & \cdots & 0 & -1 \\
-1 & 1 & 0 & 0 & \cdots & 0 \\
0 & \ddots & \ddots & \ddots & \ddots & 0 \\
0 & \cdots & -1 & 1 & 0 & 0 \\
0 & 0 & \cdots & -1 & 1 & 0 \\
0 & 0 & \cdots & 0 & -1 & 1
\end{array}\right)
$$

and it is easy to verify that $A=\sigma \sigma^{T}$ holds for both boundary conditions.

Notice that the discrete dynamics (2.12) is still conservative. Corresponding to $\mathbb{E}$ and $\mathbb{P}$ in continuous space, the phase space of the discrete dynamics is

$$
\mathbb{E}_{n}=\left\{y \mid y=\left(y_{1}, y_{2}, \cdots, y_{n}\right) \in \mathbb{R}^{n}, \frac{1}{n} \sum_{i=1}^{n} y_{i}=m\right\},
$$


and the trajectory space is $\mathbb{P}_{n}=C_{\mathbb{E}_{n}}[0,+\infty)$. We define

$$
\mathbb{E}_{n}^{0}=\left\{y \mid y=\left(y_{1}, y_{2}, \cdots, y_{n}\right) \in \mathbb{R}^{n}, \sum_{i=1}^{n} y_{i}=0\right\}=\left\{y-z \mid y, z \in \mathbb{E}_{n}\right\},
$$

which is a linear subspace of $\mathbb{R}^{n}$ with $\operatorname{rank}\left(\mathbb{E}_{n}^{0}\right)=n-1$.

2.3. Some available theoretical results. There has been some theoretical results on the dynamical analysis of the deterministic Cahn-Hilliard Equation [15, 16, 19]. For Neumann BC, stationary solutions of the deterministic dynamics are completely determined in [19]. When $f^{\prime \prime}(m)>0$, these solutions are classified into three types: the homogeneous solution $h_{0} \equiv m$, the boundary layer solutions $s_{ \pm, \kappa}^{j}$, and the transition layer solutions $i_{ \pm, \kappa}^{j}$, where $j>0$ is the index. By symmetry, $s_{+, \kappa}^{j}(x)=s_{-, \kappa}^{j}(1-x), i_{+, \kappa}^{j}(x)=i_{-, \kappa}^{j}(1-x)$; see figure 2.1(a). For larger indices $j, s_{ \pm, \kappa}^{j}, i_{ \pm, \kappa}^{j}$ are obtained from $s_{ \pm, \kappa}^{1}$ and $i_{ \pm, \kappa}^{1}$ by even reflections and rescaling [16]. When $\kappa$ becomes smaller and smaller, there are more and more solutions $s_{ \pm, \kappa}^{j}$ and $i_{ \pm, \kappa}^{j}$.

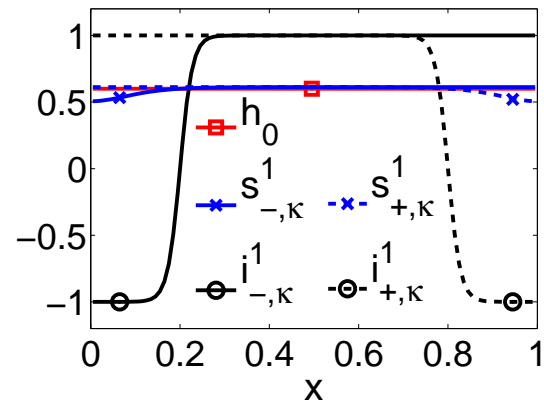

(a)

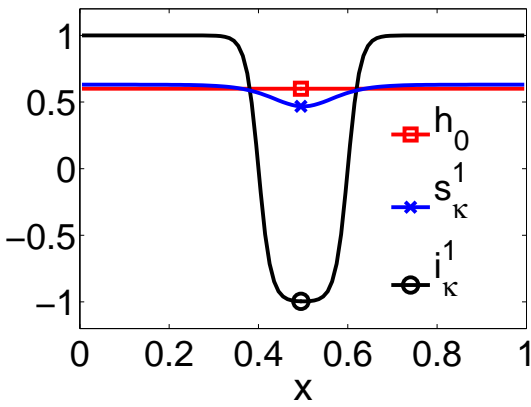

(b)

FIG. 2.1. Equilibrium states for the 1D stochastic Cahn-Hilliard dynamics (2.1). For Neumann $B C$, homogeneous solution $h_{0}$, boundary layer solutions $s_{ \pm, \kappa}^{1}$, and transition layer solutions $i_{ \pm, \kappa}^{1}$ are shown in (a). For $j>1, s_{ \pm, \kappa}^{j}$ and $i_{ \pm, \kappa}^{j}$ are obtained by even reflections and rescaling from $s_{ \pm, \kappa}^{1}$ and $i_{ \pm, \kappa}^{1}$ respectively. For periodic $B C$, homogeneous solution $h_{0}$, boundary layer solutions $s_{\kappa}^{1}$, and transition layer solutions $i_{\kappa}^{1}$ are shown in (b). For $j>1, s_{\kappa}^{j}$ and $i_{\kappa}^{j}$ are obtained by even reflections and rescaling from $s_{\kappa}^{1}$ and $i_{\kappa}^{1}$ respectively.

Among them, $h_{0}$ is locally stable and $i_{ \pm, \kappa}^{1}$ are the globally stable solutions which have the global minimum energy. In [15], the authors proved that $s_{ \pm, \kappa}^{1}$ are unstable and have a one-dimensional unstable manifold. They also conjectured that two branches of the unstable manifold connect $h_{0}$ and $i_{ \pm, \kappa}^{1}$ respectively. In fact, $s_{ \pm, \kappa}^{1}$ are the saddle points with minimal energy on $\partial \Omega\left(h_{0}\right)$, where $\Omega\left(h_{0}\right)$ denotes the attractive basin of $h_{0}$ under the deterministic version of dynamics (2.1). In [16], the authors proved that most solutions of dynamics $(2.1)$ exit $\Omega\left(h_{0}\right)$ close to the spike solutions $s_{ \pm, \kappa}^{1}$ in the zero temperature limit $\epsilon \rightarrow 0$.

The case is similar for periodic BC. Homogeneous solutions $h_{0}$, boundary layer solutions $s_{\kappa}^{j}$, and the transition layer solutions $i_{\kappa}^{j}$ exist. $i_{\kappa}^{1}$ has the global minimum energy. The spike solution $s_{\kappa}^{1}$ is unstable and has a degenerate direction as well as a one-dimensional unstable manifold; see figure 2.1(b). $s_{\kappa}^{j}$ and $i_{\kappa}^{j}$ with index $j>1$ are obtained by even reflections and rescaling from $s_{\kappa}^{1}$ and $i_{\kappa}^{1}$ respectively. 
By symmetry, we may denote $u_{a}(x)=h_{0}(x), u_{c}(x)=s_{-, \kappa}^{1}(x), u_{b}(x)=i_{-, \kappa}^{1}(x)$ for Neumann BC (shown in figure 3.1(b)), and $u_{c}(x)=s_{\kappa}^{1}(x), u_{b}(x)=i_{\kappa}^{1}(x)$ for periodic $\mathrm{BC}$ (shown in figure 3.1(c)). We will focus on numerically studying the transition from the metastable state $u_{a}(x)$ to the globally stable state $u_{b}(x)$ passing through the spike solution $u_{c}(x)$ in the zero temperature limit $\epsilon \rightarrow 0$. The computation of the optimal transition path and the transition rates are the main concern.

2.4. Nucleation behavior on large domains. Usually the nucleation dynamics on large domains can be reduced to a Poisson model for multiple droplets $[20,21]$. In this subsection we briefly discuss the nucleation behavior of the one dimensional Cahn-Hilliard dynamics on large domain. We will show that under a suitable space-time rescaling, the large domain dynamics correspond to a small $\kappa$ model on the domain $[0,1]$.

Consider $x \in[0, L]$, where $L>0$ is large. The stochastic Cahn-Hilliard equation is given by (2.1), (2.2), and (2.3) with suitable initial and boundary conditions. We make the change of variables $\tilde{x}=x / L, \tilde{t}=t / T, \tilde{u}(\tilde{x}, \tilde{t})=u(x, t)$, so that

$$
\frac{\partial u}{\partial t}=\frac{1}{T} \frac{\partial \tilde{u}}{\partial \tilde{t}}, \quad \frac{\partial u}{\partial x}=\frac{1}{L} \frac{\partial \tilde{u}}{\partial \tilde{x}},
$$

and

$$
\tilde{\eta}(\tilde{x}, \tilde{t})=\sqrt{L T} \eta(x, t), \quad \xi=\partial_{x} \eta=\frac{1}{L \sqrt{L T}} \partial_{\tilde{x}} \tilde{\eta}=\frac{1}{L \sqrt{L T}} \tilde{\xi},
$$

by the half order property of standard Wiener processes. Thus (2.1) is converted to

$$
\frac{\partial \tilde{u}}{\partial \tilde{t}}=\frac{T}{L^{2}} \frac{\partial^{2}}{\partial \tilde{x}^{2}}\left(-\frac{\kappa^{2}}{L^{2}} \frac{\partial^{2} \tilde{u}}{\partial \tilde{x}^{2}}+\tilde{u}^{3}-\tilde{u}\right)+T \sqrt{2 \epsilon} \frac{1}{L \sqrt{L T}} \tilde{\xi} .
$$

Letting $T=L^{2}, \tilde{\kappa}=\kappa / L$, we obtain

$$
\frac{\partial \tilde{u}}{\partial \tilde{t}}=\frac{\partial^{2}}{\partial \tilde{x}^{2}}\left(-\tilde{\kappa}^{2} \frac{\partial^{2} \tilde{u}}{\partial \tilde{x}^{2}}+\tilde{u}^{3}-\tilde{u}\right)+\sqrt{\frac{2 \epsilon}{L}} \xi=\frac{\partial^{2}}{\partial \tilde{x}^{2}} \frac{\delta \tilde{F}(\tilde{u})}{\delta \tilde{u}}+\sqrt{\frac{2 \epsilon}{L}} \xi,
$$

with

$$
\tilde{F}(\tilde{u})=\int_{0}^{1}\left[\frac{\tilde{\kappa}^{2}}{2}\left(\frac{\partial \tilde{u}}{\partial \tilde{x}}\right)^{2}+f(\tilde{u})\right] d \tilde{x}
$$

and

$$
\int_{0}^{1} \tilde{u}(\tilde{x}, \tilde{t}) d \tilde{x}=\frac{m}{L}=\tilde{m} .
$$

Therefore the dynamics can be reduced from $x \in[0, L]$ to $\tilde{x} \in[0,1]$ under the scaling of $\tilde{x}=x / L, \tilde{t}=t / L^{2}, \tilde{\kappa}=\kappa / L, \tilde{\epsilon}=\epsilon / L, \tilde{m}=m / L$. If $f^{\prime \prime}(\tilde{m})>0$, the system is in nucleation regime.

Notice $\tilde{\kappa} \rightarrow 0$ as $L \rightarrow+\infty$, thus the dynamics on the large domain $[0, L]$ are equivalent to the sharp interface limit of dynamics $(2.1)$ on $[0,1]$. When $\tilde{\kappa} \rightarrow 0$, the functional $\tilde{F}(u)$ becomes complicated since there are many equilibrium boundary layer solutions and transition layer solutions. The energy barrier between $u_{a}(x)$ and $u_{c}(x)$ becomes smaller, thus the transition events from $u_{a}(x)$ occur much more easily, which is in accordance with the nucleation behavior on large domain, where we are in the multiple droplet regime.

In this paper, we mainly assume that $\kappa$ is not so small and therefore confine ourselves to the single droplet regime. The numerical study of the nucleation and dynamics on large domains will be studied in the future works. 


\section{The transition path and the saddle point}

3.1. Action functional of the stochastic Cahn-Hilliard equation. Consider the 1D deterministic Cahn-Hilliard equation

$$
\frac{\partial u}{\partial t}=\frac{\partial^{2}}{\partial x^{2}} \frac{\delta F(u)}{\delta u},
$$

and suppose $a$ is a local attractor and that the basin of attraction of $a$ is defined as

$$
\Omega=\Omega(a)=\left\{w \mid \lim _{t \rightarrow+\infty} u(t)=a, u(0)=w \in \mathbb{E}\right\} .
$$

It is clear that once $u(t)$ enters $\Omega$, it won't leave the basin and will be attracted to $a$. For the stochastic dynamical system (2.1), things become different. The trajectory may escape from the basin under the perturbation of the noise (see the discussion in figure 3.1). In the case that there are two local attractors (metastable states) $a$ and $b$, as mentioned above, we are interested in the transition events between them. What is more, we want to find the most probable transition path among all the paths connecting them. Following the large deviation theory, in the zero temperature limit, the probability of the path is determined by its action functional. With physical notations, we formally have

$$
P(u \in B) \sim \int_{u \in B} \frac{1}{\mathcal{Z}} \exp \left\{-\frac{1}{\epsilon} I(u)\right\} \mathcal{D} u,
$$

where $\mathcal{Z}$ is the formal partition function, $B$ is any Borel measurable set in path space $\mathbb{P}, I$ is called the action functional, and the transition events happen along the most probable path in most cases. Denoting the probability of transition events finished before time $T$ by $P_{T}$ and by $B_{T}=\{u \mid u(0)=a, u(T)=b, u \in \mathbb{P}\}$ the set of all the possible paths, it follows that

$$
\lim _{\epsilon \rightarrow 0} \epsilon \ln P_{T}=\lim _{\epsilon \rightarrow 0} \epsilon \ln P\left(u \in B_{T}\right)=-\min _{u \in B_{T}} I(u)
$$

by large deviation theory. From this, we can see that the action functional of the dynamical system is crucial when answering Q1 and Q3 above.

For system (2.1) with periodic BC (2.5), it is shown in [17] (the action functional with Neumann BC (2.4) is similar and is given in [16]) that the action functional $I(u)$ for $u \in \mathbb{P}$ is

$$
I(u)=\frac{1}{2} I_{0}(u(0))+\int_{0}^{T} \frac{1}{4}\left\|\frac{\partial u}{\partial t}-\Delta \frac{\delta F}{\delta u}\right\|_{-1}^{2} d t,
$$

where $I_{0}$ is a good functional in $\mathbb{E},\|\rho\|_{-1}^{2}=\left\langle(-\Delta)^{-1} \rho, \rho\right\rangle, \forall \rho \in \mathbb{E}$. Notice $\Delta=\frac{\partial^{2}}{\partial x^{2}}$ in one dimension, and $(-\Delta)^{-1}$ is a bounded positive self-adjoint linear operator on $\mathbb{E}$.

Let $u(x, t) \in \mathbb{P}, a:=u(\cdot, 0), b:=u(\cdot, T)$. Suppose $\partial \Omega(a) \cap \partial \Omega(b)$ is nonempty and is called the separatrix. $u(t)$ intersects the separatrix at $t=T^{*}$. We can estimate the action functional $I(u)$ following $[22]$ :

$$
I(u)=\frac{1}{2} I_{0}(a)+\int_{0}^{T^{*}} \frac{1}{4}\left\|\frac{\partial u}{\partial t}-\Delta \frac{\delta F}{\delta u}\right\|_{-1}^{2} d t+\int_{T^{*}}^{T} \frac{1}{4}\left\|\frac{\partial u}{\partial t}-\Delta \frac{\delta F}{\delta u}\right\|_{-1}^{2} d t
$$




$$
\begin{aligned}
& =\frac{1}{2} I_{0}(a)+\int_{0}^{T^{*}} \frac{1}{4}\left\|\frac{\partial u}{\partial t}+\Delta \frac{\delta F}{\delta u}\right\|_{-1}^{2} d t \\
& \quad+\int_{T^{*}}^{T} \frac{1}{4}\left\|\frac{\partial u}{\partial t}-\Delta \frac{\delta F}{\delta u}\right\|_{-1}^{2} d t+\int_{0}^{T^{*}}\left(\frac{\partial u}{\partial t}, \frac{\delta F}{\delta u}\right) d t \\
& \geq \frac{1}{2} I_{0}(a)+(F(c)-F(a)),
\end{aligned}
$$

where $c$ is the minimizer of $F(u)$ on the separatrix (saddle point). Let $\Delta F=F(c)-$ $F(a)$ denote the energy barrier between the metastable states $a$ and $b$. From the above estimation, we obtain $I(u) \geq \frac{1}{2} I_{0}(a)+\Delta F$. On the other hand, consider a path $u^{*} \in \mathbb{P}$ which is a concatenation of two parts

$$
\lim _{t \rightarrow-\infty} u^{*}(t)=a, \lim _{t \rightarrow+\infty} u^{*}(+\infty)=c, \quad \frac{\partial u^{*}}{\partial t}=-\Delta \frac{\delta F}{\delta u}
$$

and

$$
\lim _{t \rightarrow-\infty} u^{*}(t)=c, \lim _{t \rightarrow+\infty} u^{*}(+\infty)=b, \quad \frac{\partial u^{*}}{\partial t}=\Delta \frac{\delta F}{\delta u} .
$$

Then there exists connecting paths $u$ which geometrically converge to $u^{*}$ and $I\left(u^{*}\right)=$ $\frac{1}{2} I_{0}(a)+\Delta F$ by redefining the integration interval as from $-\infty$ to $+\infty$. Thus the lower bound is obtained asymptotically. $u^{*}$ is called the minimal energy path (MEP) [7] and is the most probable transition path in the zero temperature limit. Intuitively, from (3.6) and (3.7), we know that $u^{*}$ follows the steepest ascent Cahn-Hilliard dynamics before it reaches saddle point $c$, and follows the steepest descent Cahn-Hilliard dynamics after it reaches $c$.

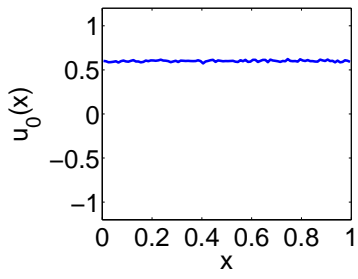

(a) initial state

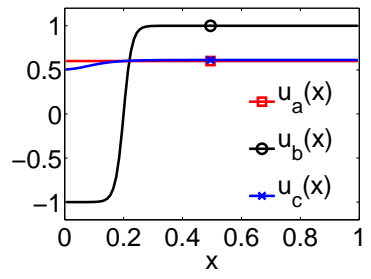

(b) Neumann BC

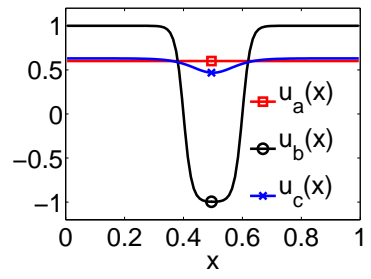

(c) periodic $\mathrm{BC}$

FIG. 3.1. Equilibrium states for $1 D$ stochastic Cahn-Hilliard dynamics (2.1) and illustration of the transition event. Starting from an arbitrary initial state $u_{0}(x)$ (shown in (a)), located in the attractive basin of the stable state $u_{a}(x)$, the system will first relax to $u_{a}(x)$ and fluctuate around $u_{a}(x)$ for a long time. Then a sudden large fluctuation of noise may drive the system to the saddle point $u_{c}(x)$, which is on the boundary of the basin of attraction. Finally, once the system escapes from the attractive basin of $u_{a}(x)$, it will evolve to another stable state $u_{b}(x)$.

3.2. Finding the minimal energy path and saddle point. The string method is an efficient method by which to study the transition path and has been applied to a wide range of problems $[7,23,24,25]$. Here, we focus on Q1 and Q2 in Section 1. Based on the action functional (3.5) and the derived MEP equation, we tailor the string method to the Cahn-Hilliard dynamics as follows. 
3.2.1. The string method algorithm. Consider a string $\varphi(s)$ in $\mathbb{P}, s \in[0,1]$ and $\varphi(0)=a, \varphi(1)=b$, where we parameterize the string using arc length instead of time. (3.6) and (3.7) are equivalent to

$$
\dot{\varphi}(s)^{\perp}=0,
$$

where $\perp$ means the projection in the direction perpendicular to $\Delta \frac{\delta F}{\delta u}$. To solve (3.8), we discretize the string $\varphi(s)$ to $\varphi_{i}, i=0,1, \cdots, N-1, N$, with $\varphi_{0}=a, \varphi_{N}=b$, and evolve $\varphi_{i}$ as in Algorithm 1 (notice that $\varphi_{i}$ is preserved in $\mathbb{E}$ by linear interpolation). The steady state of the string gives the minimal energy path.

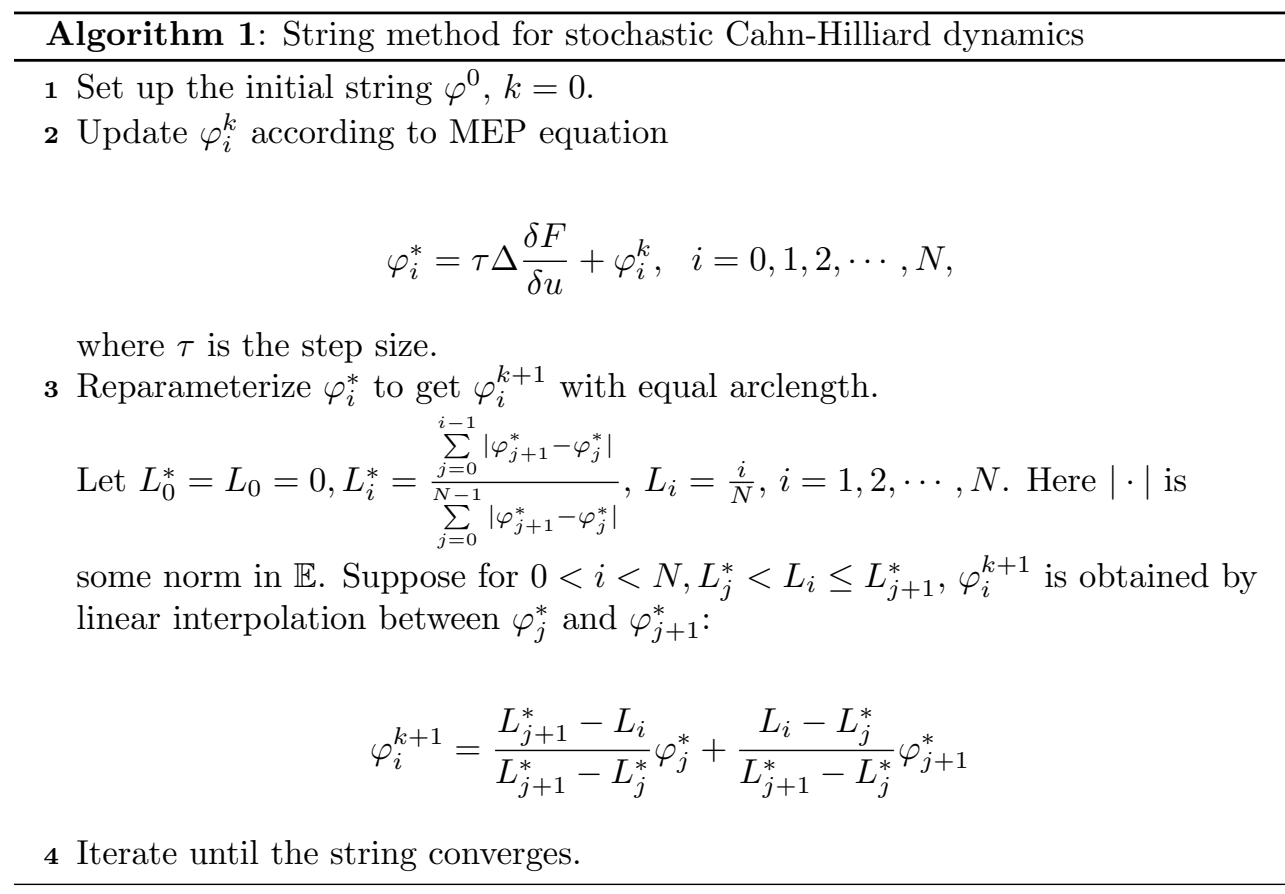

3.2.2. Tangent direction of the string at saddle point. Once $\varphi(s)$ is obtained, the saddle point $c=\varphi\left(s^{*}\right)$ and the unit tangent vector of $\varphi(s)$ at $c$ (denoted as $\vec{t}$ can be precisely calculated by using the climbing image method [8, 23]. From (3.8), we have

$$
\dot{\varphi}(s)=C(s) \frac{\partial^{2}}{\partial x^{2}} \frac{\delta F}{\delta u}(\varphi(s)),
$$

where $s$ is the arc length parameter. $C(s)$ is some scalar quantity to ensure $|\dot{\varphi}(s)|=1$. $C(s)>0$ when $s>s^{*}$ and $C(s)<0$ when $s<s^{*}$. In discrete case, expanding $\nabla V$ at $c=\varphi\left(s^{*}\right)$ and utilizing the condition of stationarity $A \nabla V(c)=0$ implies that

$$
\dot{\varphi}(s)=-C(s) \Delta s A H\left(\varphi\left(s^{*}\right)\right) \frac{\varphi(s)-\varphi\left(s^{*}\right)}{\Delta s}+O\left(\left\|\varphi(s)-\varphi\left(s^{*}\right)\right\|^{2}\right),
$$

where $H$ is the Hessian of $V$ at $c$. Letting $s>s^{*}, s \rightarrow s^{*}$, then

$$
\lim _{s \rightarrow s^{*}} \frac{\varphi(s)-\varphi\left(s^{*}\right)}{\Delta s}=\vec{t}=\dot{\varphi}\left(s^{*}\right),
$$


and thus $C(s) \Delta s$ converges. We obtain $\mu \vec{t}=A H \vec{t}$, which implies $\vec{t}$ is a unit eigenvector of $A H$ with $\mu<0$ and that the eigenvalue $\mu$ satisfies

$$
\mu=\langle\vec{t}, A H \vec{t}\rangle,
$$

where $\langle\cdot, \cdot\rangle$ denotes the inner product of vectors in $\mathbb{R}^{n}$.

3.2.3. Degeneracy of saddle point in periodic case. For Neumann BC, the two saddle points $\left(s_{-, \kappa}^{1}\right.$ and $\left.s_{+, \kappa}^{1}\right)$ are isolated. But for periodic BC (2.5), if $c:=u_{c}(x)$ is a saddle point, then an arbitrary

$$
\phi_{c}(\theta) \in S=\left\{\phi_{c}(\theta, \cdot) \mid \phi_{c}(\theta, \cdot)=u_{c}(\cdot-\theta), \theta \in[0,1]\right\}
$$

is also a saddle point and satisfies

$$
\frac{\partial^{2}}{\partial x^{2}} \frac{\delta F}{\delta u}\left(\phi_{c}(\theta)\right)=\frac{\partial^{2}}{\partial x^{2}}\left(-\kappa^{2} \frac{\partial^{2} \phi_{c}(\theta)}{\partial x^{2}}+\phi_{c}^{3}(\theta)-\phi_{c}(\theta)\right)=0 .
$$

Under periodic BC, this is equivalent to

$$
\frac{\delta F}{\delta u}\left(\phi_{c}(\theta)\right)=-\kappa^{2} \frac{\partial^{2} \phi_{c}(\theta)}{\partial x^{2}}+\phi_{c}^{3}(\theta)-\phi_{c}(\theta) \equiv C,
$$

where $C$ is constant. Taking the derivative with respect to $\theta$, we obtain

$$
\frac{\partial}{\partial \theta} \frac{\delta F}{\delta u}\left(\phi_{c}(\theta)\right)=-\kappa^{2} \frac{\partial^{2}}{\partial x^{2}} \phi_{c}^{\prime}(\theta)+\left(3 \phi_{c}^{2}(\theta)-1\right) \phi_{c}^{\prime}(\theta) \equiv 0,
$$

which indicates that

$$
\frac{\delta^{2} F}{\delta u^{2}}\left(\phi_{c}\right) \phi_{c}^{\prime}(\theta)=0
$$

Noticing that $\phi_{c}^{\prime}(\theta)=-u_{c}^{\prime}(x-\theta)$, by taking $\theta=0$ we obtain that

$$
\frac{\delta^{2} F}{\delta u^{2}}\left(u_{c}(x)\right) u_{c}^{\prime}(x)=0,
$$

which means that the Hessian at saddle point $c$ is degenerate and the degenerate direction is given by

$$
v_{d}=\frac{u_{c}^{\prime}(x)}{\left\|u_{c}^{\prime}(x)\right\|_{L^{2}}} .
$$

3.2.4. Normal direction of $\partial \Omega$ at saddle point. We consider the normal direction in discrete space and the discrete dynamics (2.12). Since $\partial \Omega$ is an invariant set of dynamics (2.12), we have

$$
A \nabla V(x) \cdot \vec{n}(x)=0, \quad \forall x \in \partial \Omega,
$$

where $\vec{n}(x)$ is normal direction of $\partial \Omega$ at $x$. Expanding $\nabla V(x)$ at saddle point $c$, and denoting $H=H(c), \vec{n}=\vec{n}(c)$, and $T_{\partial \Omega}(c)$ as the tangent space of $\partial \Omega$ at $c$, we obtain that for all $v \in T_{\partial \Omega}(c)$,

$$
\langle A H v, \vec{n}\rangle=0 \Longrightarrow\langle v, H A \vec{n}\rangle=0
$$


by the symmetry of $H$ and $A$. Together with $\langle v, \vec{n}\rangle=0$, we have

$$
H A \vec{n}=\alpha \vec{n} .
$$

Thus $A H A \vec{n}=\alpha A \vec{n}$, which means that $A \vec{n}$ is an eigenvector of $A H$. What is more, the dynamics (3.1) are unstable if perturbed along the direction $A \vec{n}$, therefor $\alpha<0$ and $A \vec{n} \| \vec{t}$. Thus we obtain

$$
\vec{n}=\frac{A^{-1} \vec{t}}{\left\|A^{-1} \vec{t}\right\|_{2}}
$$

where $\vec{n}, \vec{t} \in \mathbb{E}_{n}^{0}$ and $A^{-1}$ is well defined in $\mathbb{E}_{n}^{0}$. The fact that the tangent of the string does not coincide with the normal of $\partial \Omega$ reflects the difference between the Allen-Cahn and Cahn-Hilliard dynamics.

\section{Nucleation rate for 1D stochastic Cahn-Hilliard equation}

4.1. Nucleation rate formula. In Section 2, 1D stochastic Cahn-Hilliard Equation (2.1) is discretized to a generalized gradient system (2.12) with symmetric positive semidefinite matrix $A$. Let $a, b, c$ denote $u_{a}(x), u_{b}(x), u_{c}(x)$ and their corresponding discretized states, respectively. Now we consider the nucleation rate formula for system (2.1), (2.12) with Neumann BC (2.4) and periodic BC (2.5) (the rate formula for the Allen-Cahn equation is discussed in [20]).

4.1.1. Neumann boundary condition. Under Neumann BC (2.4), matrix $A$ has the form in (2.14) after discretization. $c$ and its reflection are two isolated saddle points as well as the energy minimizers on $\partial \Omega(a)$. In this case, setting $\epsilon_{n}=\epsilon / \Delta x$, the nucleation rate formula can be derived (see (A.37) in Appendix A for the details - the rate is doubled since two saddle points exist) as

$$
k_{n}=2 \frac{|\mu|}{2 \pi} \sqrt{\frac{\operatorname{det} H^{\dagger}(a)}{\left|\operatorname{det} H^{\dagger}(c)\right|}} e^{-\frac{\Delta V}{\epsilon_{n}}}=\frac{|\mu|}{\pi} \sqrt{\frac{\operatorname{det} H^{\dagger}(a)}{\left|\operatorname{det} H^{\dagger}(c)\right|}} e^{-\frac{\Delta F_{n}}{\epsilon}},
$$

where $\mu$ follows (3.11), and $H^{\dagger}(c)$ and $H^{\dagger}(a)$ denote the Hessian matrix confined to the subspace $\mathbb{E}_{n}^{0}$ at saddle point $c$ and metastable state $a$, respectively.

Intuitively, letting $n \rightarrow+\infty$ we obtain the nucleation rate formula for the stochastic Cahn-Hilliard Equation (2.1) with Neumann BC (2.4):

$$
k=\frac{|\mu|}{\pi}\left(\frac{\lambda_{1}^{a}}{\left|\lambda_{1}^{c}\right|}\right)^{\frac{1}{2}} \prod_{j=2}^{+\infty}\left(\lambda_{j}^{a} / \lambda_{j}^{c}\right)^{\frac{1}{2}} e^{-\frac{\Delta F}{\epsilon}},
$$

where $\mu<0$ is the unique negative eigenvalue of the operator $-\frac{\partial^{2}}{\partial x^{2}} \frac{\delta^{2} F}{\delta u^{2}}\left(u_{c}\right) . \Delta F=$ $F\left(u_{c}\right)-F\left(u_{a}\right)$ is the energy barrier. $\lambda_{j}^{a}$ are the eigenvalues of the operator $\frac{\delta^{2} F}{\delta u^{2}}\left(u_{a}\right)$ confined to $\mathbb{E}$ and satisfying

$$
0<\lambda_{1}^{a} \leq \lambda_{2}^{a} \leq \cdots \leq \lambda_{k}^{a} \leq \cdots,
$$

and $\lambda_{j}^{c}$ are the eigenvalues of operator $\frac{\delta^{2} F}{\delta u^{2}}\left(u_{c}\right)$ confined to $\mathbb{E}$ and satisfying

$$
\lambda_{1}^{c}<0<\lambda_{2}^{c} \leq \lambda_{3}^{c} \leq \cdots \leq \lambda_{k}^{c} \leq \cdots,
$$

i.e. there exists $\phi_{j}^{\gamma}(x) \in \mathbb{E}, \phi_{j}^{\gamma}(x) \not \equiv 0$ and constants $C_{j}^{\gamma} \in \mathbb{R}$ such that

$$
\frac{\delta^{2} F}{\delta u^{2}}\left(u_{\gamma}\right) \phi_{j}^{\gamma}(x)=\lambda_{j}^{\gamma} \phi_{j}^{\gamma}(x)+C_{j}^{\gamma}, \quad j=1,2, \cdots, \quad \gamma=a, c .
$$


4.1.2. Periodic boundary condition. Under periodic $\mathrm{BC}(2.5)$, matrix $A$ takes the form in (2.15) after discretization. Letting $\epsilon_{n}=\epsilon / \Delta x$, the nucleation rate formula can be derived (see (A.39) in Appendix A for the details) as

$$
k_{n}=\frac{|\mu|}{2 \pi}\left(2 \pi \epsilon_{n}\right)^{-\frac{1}{2}} \sqrt{\frac{\operatorname{det} H^{\dagger}(a)}{\left|\tilde{\operatorname{det}} H_{\dagger}^{\dagger}(c)\right|}}|| u_{c}^{\prime}\left\|_{2} e^{-\frac{\Delta V}{\epsilon_{n}}}=\frac{|\mu|}{2 \pi}(2 \pi \epsilon)^{-\frac{1}{2}} \sqrt{\frac{\operatorname{det} H^{\dagger}(a)}{\mid \tilde{\operatorname{det} H^{\dagger}(c) \mid}}}|| u_{c}^{\prime}\right\|_{L^{2}\left(\mathbb{R}^{n}\right)} e^{-\frac{\Delta F_{n}}{\epsilon}},
$$

where $\mu$ follows (3.11), det is defined as the product of all the nonzero eigenvalues of a matrix, $\|u\|_{L^{2}\left(\mathbb{R}^{n}\right)}=\left(\sum_{i=1}^{n} u_{i}^{2} \Delta x\right)^{\frac{1}{2}}=\|u\|_{2}(\Delta x)^{\frac{1}{2}}, \forall u \in \mathbb{R}^{n}$, and $H^{\dagger}(c)$ and $H^{\dagger}(a)$ denote the Hessian matrix confined to subspace $\mathbb{E}_{n}^{0}$ at saddle point $c$ and metastable state $a$ respectively.

Intuitively, letting $n \rightarrow+\infty$ we obtain the nucleation rate formula for the stochastic Cahn-Hilliard Equation (2.1) with periodic BC (2.5):

$$
k=\frac{|\mu|}{2 \pi}(2 \pi \epsilon)^{-\frac{1}{2}}\left(\frac{\lambda_{1}^{a} \lambda_{2}^{a}}{\left|\lambda_{1}^{c}\right|}\right)^{\frac{1}{2}} \prod_{j=3}^{+\infty}\left(\lambda_{j}^{a} / \lambda_{j}^{c}\right)^{\frac{1}{2}}\left\|u_{c}^{\prime}\right\|_{L^{2}} e^{-\frac{\Delta F}{\epsilon}},
$$

where all of the notations are similar to those in the Neumann boundary condition case except that $\lambda_{j}^{c}$ are the eigenvalues of $\frac{\delta^{2} F}{\delta u^{2}}\left(u_{c}\right)$ confined to $\mathbb{E}$ and satisfying

$$
\lambda_{1}^{c}<\lambda_{2}^{c}=0<\lambda_{3}^{c} \leq \cdots \leq \lambda_{k}^{c} \leq \cdots .
$$

4.2. Nucleation rate computation. Now we consider the algorithm to compute the nucleation rate according to (4.1) and (4.3). First of all, as mentioned above, $\mu$ as well as its corresponding eigenvector $\vec{t}$ and the degenerate direction $v_{d}$ (for periodic BC (2.5)) at saddle point $c$ can be obtained precisely by using the string method. For the notations, given $q \in \mathbb{R}^{n}, H \in M_{n \times n}, q^{\dagger}$ denotes the orthogonal projection of $q$ to $\mathbb{E}_{n}^{0}$ and $H^{\dagger}$ denotes the confinement of $H$ to $\mathbb{E}_{n}^{0}$, i.e. $H^{\dagger} q=(H q)^{\dagger}$ is the orthogonal projection of $H q$ to $\mathbb{E}_{n}^{0}$.

The Hessian matrix $H^{\dagger}(c)$ at saddle point $c$ has a negative eigenvalue $\lambda_{1}<0$ which appears in the term $\operatorname{det} H^{\dagger}(c)$ of (4.1) and $\tilde{\operatorname{det}} H^{\dagger}(c)$ of (4.3). By the Courant-Fischer theorem, we have

$$
\lambda_{1}=\min _{\|x\|_{2}=1, x \in \mathbb{E}_{n}^{0}}\left\langle x, H^{\dagger}(c) x\right\rangle .
$$

Based on this formula, $\lambda_{1}$ and its corresponding eigenvector $v_{1}$ can be obtained by a two step procedure similar to that in [23]. In each step, $x_{i}$ is first updated to $x_{i}^{*}$ :

$$
x_{i}^{*}=x_{i}-\Delta t H^{\dagger}(c) x_{i} .
$$

Then $x_{i}^{*}$ is projected onto the unit sphere by normalization:

$$
x_{i+1}=\frac{x_{i}^{*}}{\left\|x_{i}^{*}\right\|_{2}} .
$$

The steady state $v_{1}$ of $(4.6),(4.7)$ satisfies $H^{\dagger}(c) v_{1}=\lambda_{1} v_{1}$ and

$$
\lambda_{1}=\left\langle v_{1}, H^{\dagger}(c) v_{1}\right\rangle .
$$

Now we turn to calculate the ratio of the determinant in (4.1) and (4.3). We adopt the algorithm in $[26,24,25]$. Before describing the method, we need to modify 
$H^{\dagger}(c)$ to a symmetric positive definite matrix without changing the magnitude of its determinant. For Neumann BC, let $H_{1}^{\dagger}=H^{\dagger}(c)-2 \lambda_{1} v_{1} v_{1}^{T}$, so that the negative eigenvalue $\lambda_{1}$ of $H^{\dagger}(c)$ is transformed to an eigenvalue $-\lambda_{1}>0$ of $H_{1}^{\dagger}$ and $H_{1}^{\dagger} v_{1}=$ $-\lambda_{1} v_{1}, \operatorname{det} H_{1}^{\dagger}=\left|\operatorname{det} H^{\dagger}(c)\right|$. For periodic $\mathrm{BC}$, the zero eigenvalue also needs to be eliminated. Letting $H_{1}^{\dagger}=H^{\dagger}(c)+v_{d} v_{d}^{T}-2 \lambda_{1} v_{1} v_{1}^{T}$, we have $H_{1}^{\dagger} v_{1}=-\lambda_{1} v_{1}$, $H_{1}^{\dagger} v_{d}=v_{d}$ and $\operatorname{det} H_{1}^{\dagger}=\left|\tilde{\operatorname{det}}^{\dagger}(c)\right|$. Notice that the Hessian matrix $H^{\dagger}(a)$ is itself positive definite. Thus, we have modified the determinant ratio in formula (4.1) and (4.3) to

$$
\frac{\operatorname{det} H^{\dagger}(a)}{\operatorname{det} H_{1}^{\dagger}}
$$

which is the determinant ratio of the two symmetric positive definite matrices $H^{\dagger}(a)$ and $H_{1}^{\dagger}$.

To calculate the determinant ratio of $H^{\dagger}(a)$ and $H_{1}^{\dagger}$, define $U^{\alpha}(q)=\frac{1}{2} q^{T}\left[\alpha H_{1}^{\dagger}+\right.$ $\left.(1-\alpha) H^{\dagger}(a)\right] q$, where $q \in \mathbb{E}_{n}^{0}, \alpha \in[0,1]$, and $\beta$ is a small constant. Then

$$
Z(\alpha)=\int_{\mathbb{E}_{n}^{0}} d q \exp \left[-\beta U^{\alpha}(q)\right]=\left(2 \pi \beta^{-1}\right)^{\frac{n-1}{2}}\left\{\operatorname{det}\left[\alpha H_{1}^{\dagger}+(1-\alpha) H^{\dagger}(a)\right]\right\}^{-\frac{1}{2}} .
$$

After taking the derivative of $\ln Z(\alpha)$ with respect to $\alpha$, we have

$$
\begin{aligned}
\frac{d}{d \alpha} \ln Z(\alpha) & =\frac{1}{Z(\alpha)} \int_{\mathbb{E}_{n}^{0}} d q\left[\frac{\beta}{2} q^{T}\left(H^{\dagger}(a)-H_{1}^{\dagger}\right) q\right] \exp \left[-\beta U^{\alpha}(q)\right] \\
& =\left\langle\left[\frac{\beta}{2} q^{T}\left(H^{\dagger}(a)-H_{1}^{\dagger}\right) q\right]\right\rangle_{\alpha} \triangleq Q(\alpha),
\end{aligned}
$$

where $\langle\cdot\rangle_{\alpha}$ is the expectation with respect to

$$
\pi_{\alpha}(q)=\frac{1}{Z(\alpha)} \exp \left[-\beta U^{\alpha}(q)\right]=\frac{1}{Z(\alpha)} \exp \left\{-\frac{\beta}{2} q^{T}\left[\alpha H_{1}^{\dagger}+(1-\alpha) H^{\dagger}(a)\right] q\right\},
$$

which is an $(n-1)$-dimensional multivariate normal distribution on $\mathbb{E}_{n}^{0}$. After integration, it follows that

$$
\frac{\operatorname{det} H^{\dagger}(a)}{\operatorname{det} H_{1}^{\dagger}}=\left[\frac{Z(1)}{Z(0)}\right]^{2}=\exp \left\{2 \int_{0}^{1} Q(\alpha) d \alpha\right\}
$$

Thus the ratio can be obtained by calculating $Q(\alpha)$. More specifically, $Q(\alpha)$ is the expectation of $\frac{\beta}{2} q^{T}\left(H^{\dagger}(a)-H_{1}^{\dagger}\right) q$ under $\pi_{\alpha}(q)$. Notice that the ratio is independent of the constant $\beta$ and thus we can simply choose $\beta=1$. In our work, we evaluate this expectation by the Metropolis-Hastings method [27], which generates a Markov chain having $\pi_{\alpha}(q)$ as its stationary distribution. The reason to take the randomized algorithm is that it can be easily generalized to higher dimensions. At a state $q_{k}$ of the Markov chain, we consider two widely used proposals:

1. Random Walk (RW) proposal: $q_{*} \sim N\left(q_{k}, \sigma_{n}^{2} I_{n}\right)$,

2. Metropolis-adjusted Langevin (MALA) proposal:

$$
q_{*} \sim N\left(q_{k}+\frac{\sigma_{n}^{2}}{2} \nabla \log \pi_{\alpha}\left(q_{k}\right), \sigma_{n}^{2} I_{n}\right),
$$


where $\sigma_{n}$ is the step size. Set $q_{k+1}=q_{*}^{\dagger}$, which is the orthogonal projection of $q_{*}$ to $\mathbb{E}_{n}^{0}$ with probability

$$
a\left(q_{k}, q_{*}^{\dagger}\right)=\min \left(\frac{\pi_{\alpha}\left(q_{*}^{\dagger}\right) T_{n}\left(q_{*}^{\dagger}, q_{k}\right)}{\pi_{\alpha}\left(q_{k}\right) T_{n}\left(q_{k}, q_{*}^{\dagger}\right)}, 1\right) .
$$

Here $T_{n}(x, y) \equiv 1$ for the RW proposal and $T_{n}(x, y)=\exp \left(-\left\|z^{\dagger}\right\|_{2}^{2} / 2 \sigma_{n}^{2}\right)$ for the MALA proposal, where

$$
z=y-x-\frac{\sigma^{2}}{2} \nabla \log \pi_{\alpha}(x)
$$

\section{Numerical results}

5.1. Single droplet regime. We study the nucleation events of dynamics (2.12) from the homogeneous state $u_{a}(x) \equiv m$ to transition state $u_{b}(x)$. Both Neumann $\mathrm{BC}$ and periodic $\mathrm{BC}$ are considered. We take $n=100, \kappa=0.04, m=0.6$, and use 50 nodes along the string while using the string method, with time step size $\Delta t=5 \times 10^{-8}$. The string is parameterized using an energy weighted distance function.

For Neumann BC (2.4), we show $u_{a}(x), u_{b}(x)$, and the saddle point $u_{c}(x)$ obtained by using the string method in figure 5.1(a). The energy along the MEP is shown in figure 5.1(b). The 9th node has the maximum energy along the MEP and the energy barrier is $\Delta F=5.04 \times 10^{-5}$. More nodes are concentrated near the maximum energy node due to the utilization of the energy weighted distance function while reparameterizing the nodes. As mentioned above, after MEP is obtained by the string method, the saddle point $u_{c}(x)$ is precisely computed using climbing image method with $\left\|A \nabla V\left(u_{c}\right)\right\|_{2}=8.7 \times 10^{-10}$ and is shown in figure 5.1(a). The tangent direction $\vec{t}$ at saddle point $u_{c}(x)$ is calculated following [23], and $\mu=-3.44$ from (3.11). The outer normal direction $\vec{n}$ of $\partial \Omega$ is obtained using $\vec{n}=\frac{A^{-1} \vec{t}}{\|\left. A^{-1} \vec{t}\right|_{2}} \cdot \lambda_{1}=-1.32 \times 10^{-1}$ and $v_{1}$ are calculated using (4.6), (4.7), and (4.8). $\vec{t}, \vec{n}$ are shown in figure 5.1(c). From the profile of $\vec{t}$, we can imagine that once the dynamics passes the saddle point $u_{c}(x)$, an internal layer connecting -1 to 1 is formed [15].

For periodic BC $(2.5), u_{a}(x), u_{b}(x)$, and the saddle point $u_{c}(x)$ are shown in figure 5.1(d). The energy along the MEP is shown in figure 5.1(e). The 17 th node has the maximum energy along the MEP and the energy barrier is $\Delta F=4.48 \times 10^{-4}$. The saddle point $u_{c}(x)$ is precisely computed using the climbing image method with $\left\|A \nabla V\left(u_{c}\right)\right\|_{2}=9.8 \times 10^{-10}$ and is shown in figure 5.1(d). $\mu=-12.75$ from (3.11) and $\lambda_{1}=-1.89 \times 10^{-1} \cdot \vec{t}, \vec{n}$, and the degenerate direction $v_{d}$ are shown in figure 5.1(f).

To calculate the determinant ratio $(4.12)$, the interval $[0,1]$ for $\alpha$ is discretized into 30 subintervals and $Q(\alpha)$ is integrated using Simpson's rule. Using the RW and MALA proposals, we compute $Q(\alpha)$ for 10 runs with $2 \times 10^{6}$ iterations for each $\alpha$ in each run. Figure 5.2(a) and figure 5.2(d) show $Q(\alpha)$ as a function of $\alpha$ by taking the average of 10 runs for each $\alpha$ using the MALA proposal for Neumann BC and periodic BC respectively. The ratio obtained from $Q(\alpha)$ for 10 runs is shown in figure 5.2(b) for Neumann BC and figure 5.2(e) for periodic BC. The step size $\sigma_{n}$ is optimized with acceptance rate 0.234 for the RW proposal and 0.574 for the MALA proposal respectively, as suggested in [27]. For Neumann BC, the average ratio of the results of 10 runs is 0.4221 for the RW proposal and 0.4161 for the MALA proposal, 
with variance equal to $2.4 \times 10^{-4}$ and $6.7 \times 10^{-6}$ respectively. For periodic BC, the average ratio of the results for 10 runs is 0.0282 with the RW proposal and 0.0280 with the MALA proposal and the variance equals $2.1 \times 10^{-6}$ and $4.2 \times 10^{-8}$ respectively. These results are also shown in table 5.1. To check the results, we also calculate the eigenvalues of $H^{\dagger}(c)$ and $H^{\dagger}(a)$ directly using LAPACK software (we need to find their forms in the confined subspace $\mathbb{E}_{n}^{0}$, and they become dense matrices) and the ratio is 0.4168 for Neumann BC and 0.0282 for periodic BC. From figure 5.2(b), figure 5.2(e), and table 5.1, we conclude that Metropolis-Hastings method, with either the RW or the MALA proposal, can be used to compute $Q(\alpha)$ and the ratio. The ratio is accurate when comparing to the results obtained by directly solving the eigenvalues. We could also see that the results with the MALA proposal, which makes use of the information of $\pi_{\alpha}$, are more accurate and have smaller variance when comparing to the results with the RW proposal. For both Neumann BC and periodic BC, we show the numerical results with different $\kappa$ in table 5.2 and the nucleation rate as a function of $\epsilon$ for different $\kappa$ is plotted in figure 5.2(c), figure 5.2(f). As $\kappa$ increases, the interfacial energy will play more important roles in the total energy $F(u)$. Thus the system tends to form a flat interface and the homogeneous state $u_{a}(x)$ (no interface) become more stable. As a result, when $\kappa$ increases, the energy barrier becomes larger and nucleation rate decreases rapidly, as shown in table 5.2, figure 5.2(c), and figure $5.2(\mathrm{f})$. We also list the results for different $n$ in table 5.3. From these results, we conclude that the results are nearly the same for different $n$.

\begin{tabular}{ccccc}
\hline \hline & & Direct & RW & MALA \\
\hline \multirow{2}{*}{ Neumann } & mean & 0.4168 & 0.4221 & 0.4161 \\
& variance & & $2.4 \times 10^{-4}$ & $6.7 \times 10^{-6}$ \\
\hline \multirow{2}{*}{ periodic } & mean & 0.0282 & 0.0282 & 0.0280 \\
& variance & & $2.1 \times 10^{-6}$ & $4.2 \times 10^{-8}$ \\
\hline
\end{tabular}

TABLE 5.1. Mean and variance of the determinant ratio for 10 runs obtained using the Metropolis-Hastings method and directly computing the eigenvalues. The acceptance rates for the $R W$ and MALA proposals are 0.234 and 0.574 . Here $n=100, \kappa=0.04, m=0.6$.

\begin{tabular}{|c|c|c|c|c|c|c|c|c|}
\hline & $\kappa$ & $\lambda_{1}$ & $\mu$ & $R_{d}$ & $R_{M A L A}$ & $\Delta F_{n}$ & $\left\|u_{c}^{\prime}\right\|_{L^{2}\left(\mathbb{R}^{n}\right)}$ & $k_{n}$ \\
\hline \multirow{3}{*}{ Neumann } & 0.02 & $-1.11 \times 10^{-1}$ & -6.27 & 0.4042 & 0.4027 & $1.28 \times 10^{-5}$ & & $3.54 \times 10^{-1}$ \\
\hline & 0.03 & $-1.20 \times 10^{-1}$ & -4.16 & 0.4123 & 0.4123 & $2.67 \times 10^{-5}$ & & $5.90 \times 10^{-2}$ \\
\hline & 0.04 & $-1.32 \times 10^{-1}$ & -3.44 & 0.4168 & 0.4161 & $5.04 \times 10^{-5}$ & & $4.58 \times 10^{-3}$ \\
\hline \multirow{3}{*}{ periodic } & 0.02 & $-1.32 \times 10^{-1}$ & -13.78 & 0.0195 & 0.0194 & $5.03 \times 10^{-5}$ & 0.550 & $1.40 \times 10^{-1}$ \\
\hline & 0.03 & $-1.60 \times 10^{-1}$ & -12.41 & 0.0244 & 0.0241 & $1.57 \times 10^{-4}$ & 0.751 & $4.43 \times 10^{-6}$ \\
\hline & 0.04 & $-1.89 \times 10^{-1}$ & -12.75 & 0.0282 & 0.0280 & $4.48 \times 10^{-4}$ & 1.067 & $1.57 \times 10^{-18}$ \\
\hline
\end{tabular}

TABLE 5.2. Results with different $\kappa . \lambda_{1}$ is the negative eigenvalue of $H^{\dagger}$ at saddle point $u_{c}$. $\mu$ is the negative eigenvalue of $A H$ at saddle point $u_{c} . R_{d}$ is the determinant ratio obtained by directly solving the eigenvalues. $R_{M A L A}$ is the determinant ratio obtained by taking the average of the results of 10 runs using the Metropolis-Hastings method with the MALA proposal. $\Delta F_{n}$ is the energy barrier. $k_{n}$ is the nucleation rate. Here $\kappa=0.02,0.03,0.04, \epsilon=10^{-5}, n=100, m=0.6$.

5.2. Multiple droplet regime. To investigate the nucleation behavior on large domains, we may equivalently consider the case when $\kappa$ is small. Here we take $\kappa=0.008$, direct simulation of the discretized stochastic dynamics (2.12) is carried out with only Neumann BC (2.4) for simplicity. $n$ is taken to be 500 to capture the interface. The time step size is $\Delta t=10^{-8}$. The noise strengths $\epsilon$ are $5 \times 10^{-6}$, 


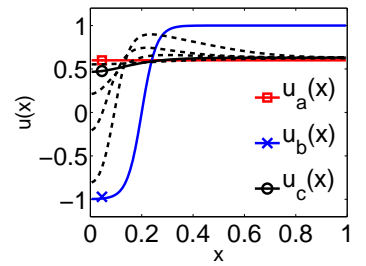

(a)

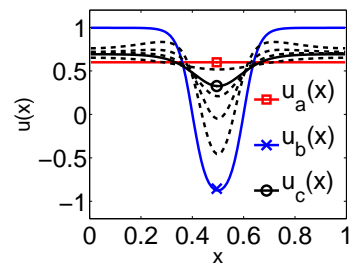

(d)

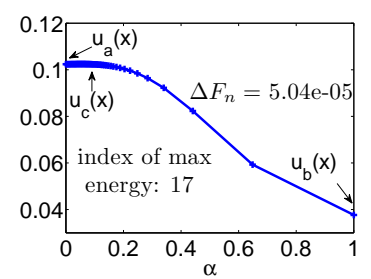

(b)

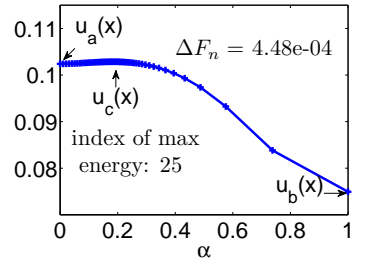

(e)

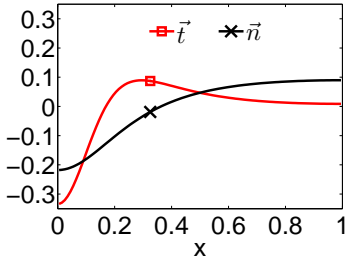

(c)

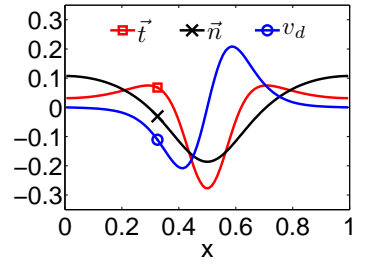

(f)

FIG. 5.1. (a) and (d): metastable states $u_{a}(x) \equiv m, u_{b}(x)$ and the saddle point $u_{c}(x)$ (solid lines), as well as several other selected states along the MEP are plotted (dashed lines). (b) and (e): Energy of nodes along MEP. (c) and (f): Tangent direction $\vec{t}$, outer normal direction $\vec{n}$ of $\partial \Omega$ at saddle point $u_{c}(x)$, degenerate direction $v_{d}$ at saddle point $u_{c}(x)$ (for periodic $B C$ ). Here $n=100, m=0.6, \kappa=0.04$. Neumann BC (2.4) is assumed in $(a)(b)(c)$ and periodic BC (2.5) is assumed in $(d)(e)(f)$.

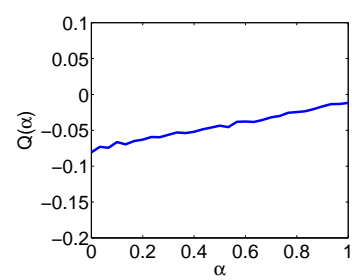

(a) $Q(\alpha)$

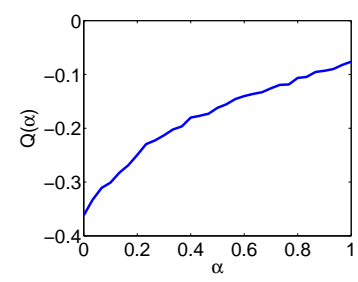

(d) $Q(\alpha)$

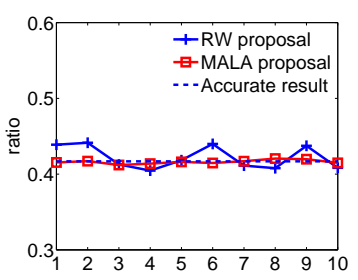

(b) Determinant ratio

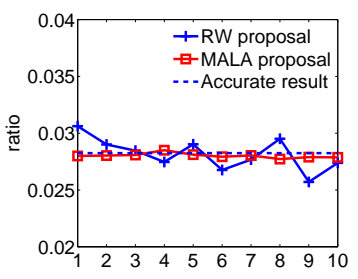

(e) Determinant ratio

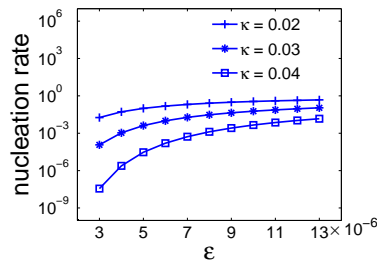

(c) Nucleation rate

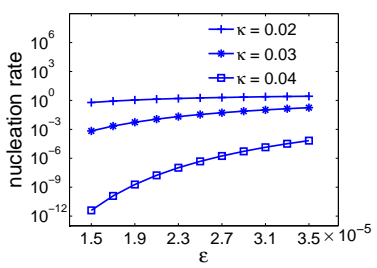

(f) Nucleation rate

FIG. 5.2. (a) and (d): $Q(\alpha)$ as a function of $\alpha$. (b) and (e): determinant ratio obtained by using the $R W$ and MALA proposals for 10 runs. $(c)$ and $(f)$ : nucleation rate for $\kappa=0.02,0.03,0.04$. $\epsilon$ ranges from $3 \times 10^{-6}$ to $1.3 \times 10^{-5}$ in (c) and from $1.5 \times 10^{-5}$ to $3.5 \times 10^{-5}$ in $(f)$. Neumann $B C$ is assumed in $(a)(b)(c)$ and periodic $B C$ is assumed in $(d)(e)(f) . n=100, m=0.6$ in all figures and $\kappa=0.04$ in $(a)(b)(d)(e)$. 


\begin{tabular}{ccccccccc}
\hline \hline & $n$ & $\lambda_{1}$ & $\mu$ & $R_{d}$ & $R_{M A L A}$ & $\Delta F_{n}$ & $\left\|u_{c}^{\prime}\right\| L^{2}\left(\mathbb{R}^{n}\right)$ & $k_{n}$ \\
\hline \multirow{5}{*}{ Neumann } & 50 & $-1.11 \times 10^{-1}$ & -6.34 & 0.4049 & 0.4007 & $1.27 \times 10^{-5}$ & $3.61 \times 10^{-1}$ \\
& 100 & $-1.11 \times 10^{-1}$ & -6.27 & 0.4042 & 0.4027 & $1.28 \times 10^{-5}$ & $3.54 \times 10^{-1}$ \\
& 150 & $-1.10 \times 10^{-1}$ & -6.26 & 0.4040 & 0.4035 & $1.28 \times 10^{-5}$ & $3.53 \times 10^{-1}$ \\
& 200 & $-1.10 \times 10^{-1}$ & -6.26 & 0.4039 & 0.4036 & $1.28 \times 10^{-5}$ & $3.53 \times 10^{-1}$ \\
& 250 & $-1.10 \times 10^{-1}$ & -6.25 & 0.4039 & 0.4039 & $1.28 \times 10^{-5}$ & $3.53 \times 10^{-1}$ \\
\hline \multirow{5}{*}{ periodic } & 50 & $-1.32 \times 10^{-1}$ & -13.96 & 0.0204 & 0.0194 & $4.98 \times 10^{-5}$ & 0.541 & $1.49 \times 10^{-1}$ \\
& 100 & $-1.32 \times 10^{-1}$ & -13.78 & 0.0195 & 0.0194 & $5.03 \times 10^{-5}$ & 0.550 & $1.40 \times 10^{-1}$ \\
& 150 & $-1.32 \times 10^{-1}$ & -13.75 & 0.0194 & 0.0192 & $5.04 \times 10^{-5}$ & 0.551 & $1.38 \times 10^{-1}$ \\
& 200 & $-1.32 \times 10^{-1}$ & -13.74 & 0.0194 & 0.0194 & $5.04 \times 10^{-5}$ & 0.552 & $1.37 \times 10^{-1}$ \\
& 250 & $-1.32 \times 10^{-1}$ & -13.74 & 0.0194 & 0.0196 & $5.04 \times 10^{-5}$ & 0.552 & $1.37 \times 10^{-1}$ \\
\hline
\end{tabular}

TABLE 5.3. Results with different $n . \lambda_{1}$ is the negative eigenvalue of $H^{\dagger}$ at saddle point $u_{c}$. $\mu$ is the negative eigenvalue of $A H$ at saddle point $u_{c} . R_{d}$ is the determinant ratio obtained by directly solving the eigenvalues. $R_{M A L A}$ is the determinant ratio obtained by taking the average of the results of 10 runs using the Metropolis-Hastings method with the MALA proposal. $\Delta F_{n}$ is the energy barrier. Here $\kappa=0.02, \epsilon=10^{-5}, m=0.6$.

$1 \times 10^{-5}$, and $3 \times 10^{-5}$ in figure 5.3, figure 5.4, and figure 5.5 respectively. From these simulation results, we can see that when $\epsilon=5 \times 10^{-6}$ is small (figure 5.3), the dynamics is in the zero temperature limit, the nucleation event is likely to happen by the boundary spike and the system will transition to the global stable transition layer state. When the noise strength $\epsilon=1 \times 10^{-5}$ is slightly larger (figure 5.4), it does not take a very long time to wait for the nucleation event to occur. Thus after the first nucleation event happens near the right boundary (figure 5.4(c)), another nucleation event occurs near the left boundary (figure 5.4(d)), while the interface formed in the first nucleation event is moving. As a result, instead of evolving to the global stable state, the system transitions to another metastable state (figure 5.4(f)). When we enlarge the noise strength $\epsilon$ to $3 \times 10^{-5}$ as in figure 5.5, the nucleation events occur more easily. Besides the two boundary spikes in figure 5.5(c), a nucleus is formed in the middle of the domain as in figure 5.5(d). Correspondingly, there are more interfaces in the final state, which has a higher energy comparing to the final state in figures 5.3(f), 5.4(f). In the later two cases, both the effects of the domain size and the finite temperature begin to play a role in the nucleation and evolution process. As a result, the nucleation events may take different paths during the transition and the system may evolve to various metastable states.

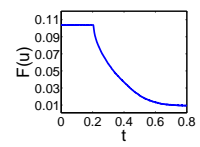

(a) $F(u)$

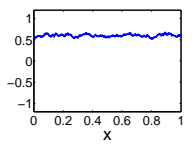

(b) $t=0.100$

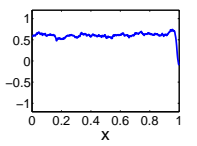

(c) $t=0.205$

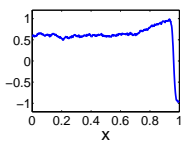

(d) $t=0.220$

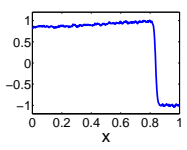

(e) $t=0.550$

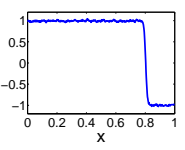

(f) $t=1.000$

Fig. 5.3. Here $\kappa=0.008, n=500, m=0.6, \epsilon=5 \times 10^{-6}$. The strength of the noise is small and therefore we are in the zero temperature limit. It takes a long time for the transition events to occur. After fluctuating around the homogeneous stable state for a long time, a spike is formed on the right boundary and the transition event occurs. Later, the interface is formed and moving from right to left. Finally, the system approaches the global stable state. 


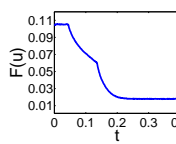

(a) $F(u)$

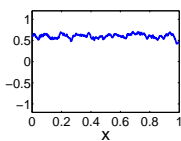

(b) $t=0.020$

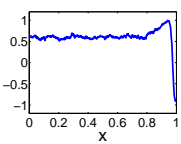

(c) $t=0.050$

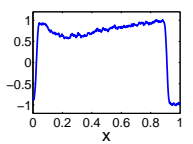

(d) $t=0.140$

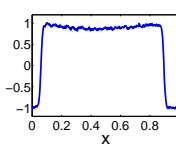

(e) $t=0.180$

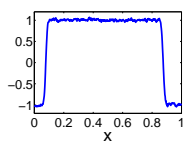

(f) $t=0.400$

FIG. 5.4. Here $\kappa=0.008, n=500, m=0.6, \epsilon=1 \times 10^{-5}$. The strength of the noise is medium and the transition events occur more easily. What is more, after the interface forms near the right boundary and moves from right to left, another transition event occurs near the left boundary and leads to the formation of another interface. As a result, the system approaches a metastable state with two interfaces near both boundaries.

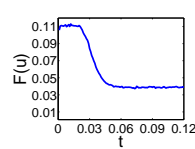

(a) $F(u)$

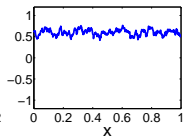

(b) $t=0.010$

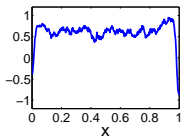

(c) $t=0.023$

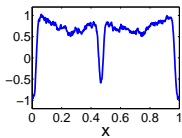

(d) $t=0.030$

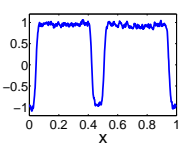

(e) $t=0.050$

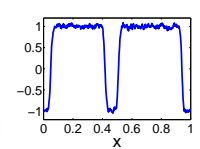

(f) $t=0.080$

FIG. 5.5. Here $\kappa=0.008, n=500, m=0.6, \epsilon=3 \times 10^{-5}$. The strength of the noise is relatively large and the transition events occur much more easily. After waiting a short time, two transition events occur on both boundaries and lead to two interfaces. Later, another transition event happens in the middle. As a result, the system approaches a metastable state containing more interfaces.

\section{Discussion}

We tailor the string method to study the transition events and compute the nucleation rate for one dimensional stochastic Cahn-Hilliard dynamics. While the Allen-Cahn equation has been studied a lot (see [20, 24, 25] and references therein), there are very few numerical works on the nucleation study of Cahn-Hilliard dynamics. Here, comparing to the previous methods for the Allen-Cahn equation, we want to emphasize the following three important aspects in our work.

- Firstly, due to the extra Laplacian operator in the Cahn-Hilliard equation, its trajectory is conservative and thus confined in a subspace. Accordingly, after discretization, unlike the Allen-Cahn equation which leads to a standard gradient system, the Cahn-Hilliard equation is converted to a generalized gradient system with a symmetric positive semidefinite coefficient matrix $A$ ( $A=I$ for the Allen-Cahn equation). Although the nucleation rate formula has been derived for a generalized gradient system with nonsingular coefficient $A$ and is applied to simple finite-dimensional cases in [22, 28], we do not find any previous works connecting it to the study of the Cahn-Hilliard equation.

- Secondly, the matrix $A$ is singular for the Cahn-Hilliard equation. To overcome this singularity, we make a change of variables to reduce the original model to a nonsingular system when deriving the nucleation rate formula (see Appendix A for more details). What is more, while calculating the rate numerically, we need to consider the determinant and eigenvalues of the Hessian matrix in a subspace.

- Thirdly, we should deal with the degeneracy of the saddle point in the periodic boundary condition case. With periodic boundary conditions, the nuclei may 
pop up from any position along the interval, thus the saddle point is no longer isolated and the potential is degenerate. A similar situation occurs for the Allen-Cahn equation with periodic boundary conditions [20].

Different from Cahn-Hilliard dynamics, in [29] the authors essentially studied the dynamics of conservative order parameters using the projected Allen-Cahn type equation

$$
\frac{\partial u}{\partial t}=\mathcal{P}\left(-\frac{\delta F}{\delta u}+\eta\right)
$$

where $\eta$ is the space-time Gaussian white noise and $\mathcal{P}$ is the orthogonal projection operator onto the confined subspace with mass conservation. As an orthogonal projection, $\mathcal{P}^{T}=\mathcal{P}$ and $\mathcal{P}^{2}=\mathcal{P}$, thus the framework proposed in this paper should be applied to these dynamics without any difficulty. Generally speaking, although these dynamics share the same metastable states and transition states with the Cahn-Hilliard dynamics studied in this paper, the trajectory of these dynamics and the transition path obtained by the string method will be different from those of the Cahn-Hilliard equation because of the difference between the orthogonal projection $\mathcal{P}$ in projected Allen-Cahn dynamics and the oblique projection $\Delta$ in Cahn-Hilliard dynamics.

We use the algorithm in $[26,24,25]$ to calculate the determinant ratio of two symmetric positive definite matrices. We adopt the Metropolis-Hastings method to compute the expectation and verify that the MALA proposal is more efficient than the RW proposal. While calculating the expectation, high dimensional integration is needed, and there are alternative algorithms to deal with this integration [30,31]. However, we should point out that, in 1D, these algorithms are more computationally expensive than directly factoring the matrix $((n-1)$-dimensional matrix $)$ to get eigenvalues. We also want to mention that ratios of functional determinants arises in several other situations. Calculating the ratio of two functional determinants is a general topic and interesting theoretical results have been obtained in some special cases (see $[32,33]$ for example). We will investigate the possibility to utilize their results and methods in solving our problem in the future.

In this work, we mainly focus on the single droplet regime in the zero temperature limit. However, in reality, we need to consider nucleation on large domains $(\kappa \rightarrow 0)$ in finite temperature, in which case the dynamics are much more complicated and interesting, as the direct simulation results show. For the case of large domains, the dynamics are in the multiple droplet regime and can be connected with the Poisson model and KJMA dynamics; see [20, 21] for detailed discussions. For the case of finite temperature, instead of finding the most probable transition path, transition tube theory and the transition path theory may need to be considered [34].

Future study will be concentrated on higher dimensional stochastic Cahn-Hilliard equations, models with more complex energy forms and the nucleation events on large domains. More efficient algorithms for calculating the ratio of matrix determinants will also be investigated.

\section{Conclusions}

In this paper, we focus on numerically studying the transition events of the stochastic Cahn-Hilliard equation. We tailor the string method for computing the most probable transition path in the zero temperature limit based on large deviation theory. We derive the nucleation rate formula for stochastic Cahn-Hilliard dynamics through finite dimensional discretization. Both Neumann boundary condition and periodic boundary condition are considered. After discretization, we obtain generalized 
gradient dynamics with a degenerate potential and confined trajectory. We discuss the algorithmic issues for calculating the nucleation rate, especially high dimensional sampling for computing the determinant ratios. The methods presented in the current paper are generally for Cahn-Hilliard dynamics with conservation for the order parameters. Application of the methods to more complex and higher dimensional problems will be discussed in continued publications.

Acknowledgments. T. Li acknowledges the support from NSFC under grants 11171009 and 91130005. P. Zhang acknowledges support from NSFC under grant 50930003.

Appendix A. Nucleation rate formula for 1D stochastic Cahn-Hilliard equation. In this section, we focus on Q3 in Section 1 and derive the nucleation rate formula for

$$
d X_{t}=-A \nabla V\left(X_{t}\right) d t+\sqrt{2 \epsilon} \sigma d W_{t}, \quad A=\sigma \sigma^{T},
$$

which is related to the discrete version (2.12) of the 1D stochastic Cahn-Hilliard Equation (2.1). The case when $A=I$ is studied for a nondegenerate potential in [22] and for a degenerate potential in [20], and the case for general positive definite matrix $A$ for a nondegenerate potential is studied in $[22,28]$. Here we adapt their derivations for a symmetric positive semidefinite matrix $A$ with degenerate potential.

A.1. Mean first exit time problem when $A$ is symmetric and positive definite. Assume matrix $A$ is symmetric and positive definite. The semi-definite case will be deferred to A.3. Consider the deterministic system

$$
d X_{t}=-A \nabla V\left(X_{t}\right) d t
$$

and suppose $x_{0}$ is a local attractor of it and that $\Omega=\Omega\left(x_{0}\right)$ is the corresponding basin of attraction. For stochastic system (A.1), starting from $X_{0} \in \Omega, X_{t}$ may escape from $\Omega$. In this situation,

$$
\tau(x)=\inf _{t}\left\{t \mid X_{t} \notin \Omega, X_{0}=x \in \Omega\right\}
$$

tells, under the perturbation of noise, the first time when the trajectory leaves the basin of attraction, and its expectation $E \tau(x)$ is called mean first exit time and shows how long in average the system will stay in $\Omega$ if starting from $x \in \Omega$.

Using Ito's formula, for any smooth function $g(x), x \in \mathbb{R}^{n}$,

$$
d g\left(X_{t}\right)=\nabla g \cdot d X_{t}+\frac{1}{2} d X_{t}^{T} \nabla^{2} g d X_{t}=L_{\epsilon} g d t+\sqrt{2 \epsilon} \nabla g \cdot\left(\sigma d W_{t}\right),
$$

where $L_{\epsilon} g=\epsilon A: \nabla^{2} g-(A \nabla V) \cdot \nabla g$ is the backward Kolmogorov operator, and the contraction of two tensors is denoted by $A: B=\sum_{i, j} a_{i j} b_{i j}$. We take a special $g(x)$ which satisfies

$$
\begin{cases}L_{\epsilon} g=-1, & x \in \Omega \\ g=0, & x \in \partial \Omega\end{cases}
$$

For $g$ satisfying (A.4), integrate (A.3) on both sides from $t=0$ to $\tau(x)$ and take the expectation, we conclude that $g(x)=E \tau(x)$ by noticing that the stochastic integral 
vanishes. Consider $w(x)=e^{-\frac{K}{\epsilon}} g(x)$, where $K$ is a constant to be determined later. From (A.4), $w(x)$ satisfies

$$
\begin{cases}L_{\epsilon} w=-e^{-\frac{K}{\epsilon}}, & x \in \Omega, \\ w=0, & x \in \partial \Omega .\end{cases}
$$

Far from $\partial \Omega$, we consider the expansion $w(x)=\sum_{i=0}^{\infty} w_{i}(x) \epsilon^{i}$, substitute it into (A.5) and compare the coefficients; we obtain the equation for leading term

$$
-(A \nabla V) \cdot \nabla w_{0}=0,
$$

which indicates that $w_{0}(x)$ is constant along (A.2). Since $x_{0}$ is the attractor of (A.2), we obtain

$$
w_{0}(x) \equiv w_{0}\left(x_{0}\right)=C_{0} .
$$

On the other hand, the invariant distribution $p(x)=\frac{1}{Z} e^{-\frac{V(x)}{\epsilon}}$, where $Z$ is a normalized constant, satisfies the Fokker-Planck equation

$$
\epsilon \nabla \cdot(A \nabla p)+\nabla \cdot(p A \nabla V)=0 .
$$

Multiply (A.5) by $p(x)$ and integrate over $\Omega$; by using (A.7) and integration by parts we obtain

$$
\int_{\partial \Omega} \epsilon e^{-\frac{V(x)}{\epsilon}}(A \nabla w) \cdot \vec{n} d \sigma=-e^{-\frac{K}{\epsilon}} \int_{\Omega} e^{-\frac{V(x)}{\epsilon}} d x
$$

where $\vec{n}$ is the outer normal of $\partial \Omega$.

To simplify the LHS of (A.8), we study $w$ near the boundary $\partial \Omega$. For simplicity, we parameterize $\Omega$ by $x=\left(x_{1}, x_{2}, \cdots, x_{n}\right)^{T}$ with $x_{n}=\operatorname{dist}(x, \partial \Omega)$ near $\partial \Omega$, so that $\vec{n}=(0,0, \cdots, 0,-1)^{T}$. We introduce the variable $y=B x$, where

$$
B=\left(\begin{array}{cccccc}
1 & 0 & 0 & \cdots & 0 & -\frac{a_{n 1}}{a_{n n}} \\
0 & 1 & 0 & \cdots & 0 & -\frac{a_{n 2}}{a_{n n}} \\
0 & 0 & 1 & 0 & \cdots & -\frac{a_{n 3}}{a_{n n}} \\
0 & 0 & \ddots & \ddots & 0 & \vdots \\
0 & 0 & \cdots & 0 & 1 & -\frac{a_{n n-1}}{a_{n n}} \\
0 & 0 & \cdots & 0 & 0 & \frac{1}{a_{n n}}
\end{array}\right) .
$$

Then $\nabla_{x}=B^{T} \nabla_{y}$ holds by the chain rule. Here $\nabla_{x}, \nabla_{y}$ are the gradient operators with respect to variables $x$ and $y$, respectively. With this new variable $y$, (A.5) is transformed to

$$
\begin{cases}\epsilon\left(B A B^{T}\right): \nabla_{y}^{2} w-\nabla_{y} V \cdot\left(B A B^{T} \nabla_{y} w\right)=-e^{-\frac{K}{\epsilon}}, & y \in \Omega_{y}, \\ w=0, & y \in \partial \Omega_{y},\end{cases}
$$

where $\Omega_{y}$ is the image of $\Omega$ in coordinate $y$. It is easy to verify that

$$
\frac{\partial w}{\partial y_{n}}=\sum_{i=1}^{n} a_{n i} \frac{\partial w}{\partial x_{i}}=-(A \nabla w) \cdot \vec{n} .
$$


We change the variable again by stretching the $n$th component $y_{n}$ of $y$ to $z=y_{n} / \sqrt{\epsilon}$ and let $C=B A B^{T}=\left(c_{i j}\right)$. Direct calculation shows that $c_{n n}=1 / a_{n n}>0$, and $c_{i n}=c_{n i}=0$ for $i \neq n$. What is more, since $\partial \Omega$ is the invariant set of the dynamics (A.2), we conclude

$$
\left.\frac{\partial V}{\partial y_{n}}\right|_{y_{n}=0}=\left.\frac{\partial V}{\partial y_{n}}\right|_{\partial \Omega}=\left.\sum_{i=1}^{n} a_{n i} \frac{\partial V}{\partial x_{i}}\right|_{\partial \Omega}=-\left.(A \nabla V) \cdot \vec{n}\right|_{\partial \Omega}=0 .
$$

From (A.9), it follows that

$$
\frac{\partial^{2} w}{\partial z^{2}}+z k\left(y^{\prime}\right) \frac{\partial w}{\partial z}+L\left(y^{\prime}\right) w+O(\sqrt{\epsilon})=-a_{n n} e^{-\frac{K}{\epsilon}}
$$

where

$$
\begin{aligned}
y^{\prime} & =\left(y_{1}, y_{2}, \cdots, y_{n-1}\right), \\
k\left(y^{\prime}\right) & =-\frac{\partial^{2} V\left(y^{\prime}, 0\right)}{\partial y_{n}^{2}}=-\sum_{i, j=1}^{n} a_{n i} a_{n j} \frac{\partial^{2} V}{\partial x_{i} \partial x_{j}}=-\langle A H A \vec{n}, \vec{n}\rangle, \\
L\left(y^{\prime}\right) & =-a_{n n} \sum_{j=1}^{n-1}\left(\sum_{i=1}^{n-1} c_{i j} \frac{\partial V\left(y^{\prime}, 0\right)}{\partial y_{i}}\right) \frac{\partial}{\partial y_{j}} .
\end{aligned}
$$

Expanding $w(x)=\sum_{i=0}^{\infty} w_{i}(x) \epsilon^{i}$ again, substituting it into (A.11), and comparing the coefficients, we obtain

$$
\frac{\partial^{2} w_{0}}{\partial z^{2}}+z k\left(y^{\prime}\right) \frac{\partial w_{0}}{\partial z}+L\left(y^{\prime}\right) w_{0}=0 .
$$

Thus we obtain the equation for $w_{0}$ as a function of a single variable $z$, which can be integrated as

$w_{0}\left(y^{\prime}, z\right)=C_{1}\left(y^{\prime}\right) \int_{0}^{z} e^{-\frac{1}{2} k\left(y^{\prime}\right)} \eta^{2} d \eta-\int_{0}^{z} e^{-\frac{1}{2} k\left(y^{\prime}\right) \eta^{2}}\left[\int_{0}^{\eta} e^{\frac{1}{2} k\left(y^{\prime}\right) s^{2}} L\left(y^{\prime}\right) w_{0}\left(y^{\prime}, s\right) d s\right] d \eta$,

with

$$
\frac{\partial w_{0}}{\partial z}\left(y^{\prime}, 0\right)=C_{1}\left(y^{\prime}\right)
$$

Letting $z \rightarrow+\infty$, we obtain

$$
w_{0}\left(y^{\prime},+\infty\right)=C_{1}\left(y^{\prime}\right) \sqrt{\frac{\pi}{2 k\left(y^{\prime}\right)}}-C_{2}\left(y^{\prime}\right)
$$

where

$$
C_{2}\left(y^{\prime}\right)=\int_{0}^{+\infty} e^{-\frac{1}{2} k\left(y^{\prime}\right) \eta^{2}}\left[\int_{0}^{\eta} e^{\frac{1}{2} k\left(y^{\prime}\right) s^{2}} L\left(y^{\prime}\right) w_{0}\left(y^{\prime}, s\right) d s\right] d \eta .
$$

Together with (A.15) and (A.6), we have

$$
C_{1}\left(y^{\prime}\right)=\left(C_{0}+C_{2}\left(y^{\prime}\right)\right) \sqrt{\frac{2 k\left(y^{\prime}\right)}{\pi}} .
$$


From (A.14), on $\partial \Omega$ we have

$$
-\left(A \nabla_{x} w\right) \cdot \vec{n}=\frac{\partial w}{\partial y_{n}}=\epsilon^{-\frac{1}{2}} \frac{\partial w}{\partial z}=\epsilon^{-\frac{1}{2}} C_{1}\left(y^{\prime}\right) .
$$

Using (A.17), (A.8) can be simplified to give

$$
\epsilon^{\frac{1}{2}} \int_{\partial \Omega} e^{-\frac{V(x)}{\epsilon}}\left(C_{0}+C_{2}\left(y^{\prime}\right)\right) \sqrt{\frac{2 k\left(y^{\prime}\right)}{\pi}} d \sigma=e^{-\frac{K}{\epsilon}} \int_{\Omega} e^{-\frac{V(x)}{\epsilon}} d x .
$$

Suppose that $c \in \partial \Omega$ is the saddle point of $V(x)$ on $\partial \Omega$, and its corresponding coordinate in $y$ is $\left(y_{*}^{\prime}, 0\right)$. Using Laplace's method, and noticing that $C_{2}\left(y_{*}^{\prime}\right)=0$ since $\nabla_{x} V(c)=0$, we obtain

$\epsilon^{\frac{1}{2}} \int_{\partial \Omega} e^{-\frac{V(x)}{\epsilon}}\left(C_{0}+C_{2}\left(y^{\prime}\right)\right) \sqrt{\frac{2 k\left(y^{\prime}\right)}{\pi}} d \sigma=\epsilon^{\frac{1}{2}}\left[C_{0} \sqrt{\frac{2 k\left(y_{*}^{\prime}\right)}{\pi}}+O(\epsilon)\right] \sqrt{\frac{(2 \pi \epsilon)^{n-1}}{\operatorname{det} H^{\perp}(c)}} e^{-\frac{V(c)}{\epsilon}}$,

where $H^{\perp}$ denotes the Hessian matrix of $V(x)$ constrained to $\partial \Omega$. For the RHS of (A.18), let $a=x_{0}$ denote the attractor of $V(x)$ which minimizes $V(x)$ in $\Omega$; using Laplace's method, we have

$$
e^{-\frac{K}{\epsilon}} \int_{\Omega} e^{-\frac{V(x)}{\epsilon}} d x=\sqrt{\frac{(2 \pi \epsilon)^{n}}{\operatorname{det} H(a)}} e^{-\frac{V(a)+K}{\epsilon}}(1+O(\epsilon)) .
$$

From (A.19) and (A.20), we obtain $K=V(c)-V(a)=\Delta V$ and

$$
C_{0}=\pi \sqrt{\frac{\operatorname{det} H^{\perp}(c)}{k\left(y_{*}^{\prime}\right) \operatorname{det} H(a)}} .
$$

When $\epsilon$ is small, $w(x) \approx w_{0}(x)=C_{0}$, the mean first exit time is

$$
E \tau(x)=w(x) e^{\frac{K}{\epsilon}} \approx C_{0} e^{\frac{K}{\epsilon}}=\pi \sqrt{\frac{\operatorname{det} H^{\perp}(c)}{k\left(y_{*}^{\prime}\right) \operatorname{det} H(a)}} e^{\frac{\Delta V}{\epsilon}} .
$$

The nucleation rate is given by

$$
k=\frac{1}{2 E \tau(x)}=\frac{1}{2 \pi} \sqrt{\frac{k\left(y_{*}^{\prime}\right) \operatorname{det} H(a)}{\operatorname{det} H^{\perp}(c)}} e^{-\frac{\Delta V}{\epsilon}}=\frac{|\langle A H A \vec{n}, \vec{n}\rangle|^{1 / 2}}{2 \pi} \sqrt{\frac{\operatorname{det} H(a)}{\operatorname{det} H^{\perp}(c)}} e^{-\frac{\Delta V}{\epsilon}},
$$

which is in accordance with the result in [22] using Kramer's method.

It is not convenient to obtain the nucleation rate numerically using formula (A.23) since we need to compute the determinant of $H(c)$ confined to the subspace $\partial \Omega$, which is not explicitly known. For this reason, we follow [28] to derive another form of the rate formula. At saddle point $c$, the outer normal of $\partial \Omega$ satisfies $\vec{n}=\frac{A^{-1} \vec{t}}{\|\left. A^{-1} \vec{t}\right|_{2}}=$ $-\frac{H(c) \vec{t}}{\|H(c) \vec{t}\|_{2}}$, and $k\left(y_{*}^{\prime}\right)=-\frac{\mu \vec{t} \cdot \vec{n}}{\left\|A^{-1} \vec{t}\right\|_{2}}$. Reconsidering the LHS of (A.18), we have

$$
\epsilon^{\frac{1}{2}} \int_{\partial \Omega} e^{-\frac{V(x)}{\epsilon}}\left(C_{0}+C_{2}\left(y^{\prime}\right)\right) \sqrt{\frac{2 k\left(y^{\prime}\right)}{\pi}} d \sigma
$$




$$
\begin{aligned}
& =\epsilon^{\frac{1}{2}}\left(C_{0}+O(\epsilon)\right) \int_{\partial \Omega} e^{-\frac{V(x)}{\epsilon}} \sqrt{\frac{2 k\left(y_{*}^{\prime}\right)}{\pi}} d \sigma \\
& \approx \frac{C_{0}}{\pi} e^{-\frac{V(c)}{\epsilon}} \int_{\partial \Omega} \int_{-\infty}^{+\infty}|\mu|(\vec{n} \cdot \vec{t}) e^{-\frac{1}{2 \epsilon}\left(\langle x-c, H(c)(x-c)\rangle+\frac{\mu^{2}(\vec{n} \cdot \vec{t})^{2}}{k\left(y_{*}^{\prime}\right)} s^{2}\right)} d \sigma d s \\
& =\frac{C_{0}}{\pi}|\mu| e^{-\frac{V(c)}{\epsilon}} \int_{\mathbb{R}^{n}} e^{-\frac{1}{2 \epsilon}(\langle x-c, \tilde{H}(c)(x-c)\rangle)} d x \\
& =\frac{C_{0}}{\pi}|\mu| e^{-\frac{V(c)}{\epsilon}} \frac{(2 \pi \epsilon)^{\frac{n}{2}}}{(\operatorname{det} \tilde{H}(c))^{\frac{1}{2}}} .
\end{aligned}
$$

In (A.24), we make the decomposition $x=c+x_{1}+s \vec{t}, x_{1} \perp \vec{n}, s \in \mathbb{R}$ and the following change of variables: $\tilde{H}=\tilde{H}(c)$ is given by

$$
\tilde{H}=H(c)\left(I-2 \frac{\vec{t} \vec{n}^{T}}{\vec{t} \cdot \vec{n}}\right)=H(c) \tilde{I},
$$

where $\tilde{I}=I-2 \vec{t} \vec{n}^{T} /(\vec{t} \cdot \vec{n})$. Direct calculation shows

$$
\langle x-c, \tilde{H}(x-c)\rangle=\left\langle x_{1}, H(c) x_{1}\right\rangle+\frac{\mu^{2}(\vec{n} \cdot \vec{t})^{2}}{k\left(y_{*}^{\prime}\right)} s^{2}
$$

and $\tilde{H}^{T}=\tilde{H}$. From the definition of $\tilde{I}$, we obtain $\tilde{I} v=v, \forall v \perp \vec{n}$, and $\tilde{I} \vec{t}=-\vec{t}$, thus $\operatorname{det} \tilde{I}_{\theta}=-1$ and

$$
\operatorname{det} \tilde{H}=-\operatorname{det} H(c)=|\operatorname{det} H(c)| .
$$

Substituting this into (A.24), we obtain

$$
\epsilon^{\frac{1}{2}} \int_{\partial \Omega} e^{-\frac{V(x)}{\epsilon}}\left(C_{0}+C_{2}\left(y^{\prime}\right)\right) \sqrt{\frac{2 k\left(y^{\prime}\right)}{\pi}} d \sigma=\frac{C_{0}}{\pi}|\mu| e^{-\frac{V(c)}{\epsilon}} \frac{(2 \pi \epsilon)^{\frac{n}{2}}}{|\operatorname{det} H(c)|^{\frac{1}{2}}} .
$$

Together with (A.20), we obtain the nucleation rate

$$
k=\frac{|\mu|}{2 \pi} \sqrt{\frac{\operatorname{det} H(a)}{|\operatorname{det} H(c)|}} e^{-\frac{\Delta V}{\epsilon}} .
$$

A.2. Nucleation rate formula when the potential is degenerate. We follow [20] to consider the rate formula for (A.1) when the potential $V(x)$ is degenerate on $\partial \Omega$. Suppose the minimizers of $V(x)$ on $\partial \Omega$ can be parameterized as

$$
S=\left\{\gamma(\theta) \mid \theta \in[0,1]^{d}\right\},
$$

and $V(\gamma(\theta))=V_{s}=\min _{x \in \partial \Omega} V(x)$. In this case, we can not directly apply Laplace's method to the LHS of (A.18). Notice that the major contribution to the LHS of (A.18) comes from $\gamma(\theta)$, and $C_{2}\left(y^{\prime}\right)=0$ at $\gamma(\theta)$. Let $\vec{t}(\theta)$ denote the eigenvector of $A H(\theta)$ corresponding to the negative eigenvalue $\mu(\theta)<0$, where $H(\theta)=H(\gamma(\theta))$ is the Hessian matrix of $V(x)$ at $\gamma(\theta)$. Then following the notation of A.1, the outer normal direction of $\partial \Omega$ satisfies $\vec{n}(\theta)=\frac{A^{-1} \vec{t}(\theta)}{\left\|A^{-1} \vec{t}(\theta)\right\|_{2}}=-\frac{H(\theta) \vec{t}(\theta)}{\|H(\theta) \vec{t}(\theta)\|_{2}}$, and $k\left(y^{\prime}\right)=-\frac{\mu(\theta) \vec{t}(\theta) \cdot \vec{n}(\theta)}{\left\|A^{-1} \vec{t}(\theta)\right\|_{2}}$. Let $P x=$ $\gamma(\theta)$ denote the orthogonal projection of $x$ on $S$ and $O(\theta)=\{P x=\gamma(\theta), x \in \partial \Omega\}$. 
For the LHS of (A.18), applying the Co-Area formula as in [20], omitting the higher term of $\epsilon$, and adopting similar methods as in A.1, we have

$$
\begin{aligned}
& \epsilon^{\frac{1}{2}} \int_{\partial \Omega} e^{-\frac{V(x)}{\epsilon}}\left(C_{0}+C_{2}\left(y^{\prime}\right)\right) \sqrt{\frac{2 k\left(y^{\prime}\right)}{\pi}} d \sigma \\
= & \epsilon^{\frac{1}{2}} \int_{[0,1]^{d}} \int_{O(\theta)} J(\theta) e^{-\frac{V(x)}{\epsilon}}\left(C_{0}+C_{2}\left(y^{\prime}\right)\right) \sqrt{\frac{2 k\left(y^{\prime}\right)}{\pi}} d \sigma^{\prime} d \theta \\
= & \frac{C_{0}}{\pi} e^{-\frac{V_{s}}{\epsilon}} \int_{[0,1]^{d}} J(\theta) \int_{O(\theta)} \int_{-\infty}^{+\infty}\{|\mu(\theta)|(\vec{n}(\theta) \cdot \vec{t}(\theta)) \\
& \left.\times e^{-\frac{1}{2 \epsilon}\left(\langle x-P x, H(P x)(x-P x)\rangle+\frac{\mu^{2}(\theta)(\vec{n}(\theta) \cdot \vec{t}(\theta))^{2}}{k\left(y^{\prime}\right)} s^{2}\right)}\right\} d \sigma^{\prime} d s d \theta \\
= & \frac{C_{0}}{\pi} e^{-\frac{V_{s}}{\epsilon}} \int_{[0,1]^{d}} \int_{\{P x=\gamma(\theta)\}} J(\theta)|\mu(\theta)| e^{-\frac{1}{2 \epsilon}(\langle x-P x, \tilde{H}(P x)(x-P x)\rangle)} d H^{n-d} d \theta \\
= & \frac{C_{0}}{\pi} e^{-\frac{V_{s}}{\epsilon}} \int_{[0,1]^{d}} J(\theta)(2 \pi \epsilon)^{(n-d) / 2} \frac{|\mu(\theta)|}{\left(\operatorname{det} \tilde{H}_{\theta}(\theta)\right)^{\frac{1}{2}}} d \theta .
\end{aligned}
$$

In (A.28), $J(\theta)$ is the Jacobian defined as

$$
J(\theta)=\sqrt{\operatorname{det}\left(\nabla \gamma(\theta)(\nabla \gamma(\theta))^{T}\right)},
$$

and $\sigma^{\prime}$ is the volume element on $O(\theta)$. We have made the decomposition $x=P x+$ $x_{1}+s \vec{t}(\theta), x_{1} \perp \vec{n}(\theta), s \in \mathbb{R}$ and the following change of variables: $\tilde{H}(\theta)=\tilde{H}(\gamma(\theta))$ is given by

$$
\tilde{H}(\theta)=H(\theta)\left(I-2 \frac{\vec{t}(\theta) \vec{n}(\theta)^{T}}{\vec{t}(\theta) \cdot \vec{n}(\theta)}\right)=H(\theta) \tilde{I}(\theta),
$$

where $\tilde{I}(\theta)=I-2 \frac{\vec{t}(\theta) \vec{n}(\theta)^{T}}{\vec{t}(\theta) \cdot \vec{n}(\theta)}$. $\tilde{H}_{\theta}(\theta)$ is $\tilde{H}(\theta)$ confined to $\left\{x \mid P x=\gamma(\theta), x \in \mathbb{R}^{n}\right\}$. Direct calculation shows that

$$
\langle x-P x, \tilde{H}(\theta)(x-P x)\rangle=\left\langle x_{1}, H(\theta) x_{1}\right\rangle+\frac{\mu(\theta)^{2}(\vec{n}(\theta) \cdot \vec{t}(\theta))^{2}}{k\left(y^{\prime}\right)} s^{2},
$$

and $\tilde{H}^{T}(\theta)=\tilde{H}(\theta)$.

Consider $\mathbb{R}^{n}=M(\theta) \oplus M(\theta)^{\perp}, M(\theta)=\left\{v \mid H(\theta) v=0, v \in \mathbb{R}^{n}\right\}, \operatorname{rank}(M(\theta))=d$. For $v \in M(\theta)$,

$$
v \cdot \vec{n}(\theta)=-v \cdot \frac{H(\theta) \vec{t}(\theta)}{|H(\theta) \vec{t}(\theta)|}=-\frac{(H(\theta) v) \cdot \vec{t}(\theta)}{|H(\theta) \vec{t}(\theta)|}=0,
$$

which indicates $\vec{n}(\theta) \in M(\theta)^{\perp}$. Suppose

$$
M(\theta)^{\perp}=\operatorname{span}\left\{\vec{n}(\theta), v_{1}(\theta), v_{2}(\theta), \cdots, v_{n-d-1}(\theta)\right\},
$$

where $v_{i}(\theta) \perp \vec{n}(\theta), i=1,2, \cdots, n-d-1$. Let $H_{\theta}(\theta), \tilde{I}_{\theta}(\theta)$ denote the constraint of $H(\theta)$ and $\tilde{I}(\theta)$ in $M(\theta)^{\perp}$, respectively. It is clear that $\tilde{H}_{\theta}(\theta)=H_{\theta}(\theta) \tilde{I}_{\theta}(\theta)$ and $\tilde{I}(\theta) v_{i}(\theta)=v_{i}(\theta)$, since $v_{i}(\theta) \perp \vec{n}(\theta)$. Consider $\vec{t}(\theta)=t_{1}+t_{2}, t_{1} \in M(\theta), t_{2} \in M(\theta)^{\perp}$, then

$$
\tilde{I}(\theta) \vec{t}(\theta)=-\vec{t}(\theta), t_{1} \perp \vec{n}(\theta) \Longrightarrow \tilde{I}(\theta) t_{2}=-2 t_{1}-t_{2}
$$


Thus we obtain $\tilde{I}_{\theta}(\theta) v_{i}(\theta)=v_{i}(\theta), \tilde{I}_{\theta}(\theta) t_{2}=-t_{2}$, which indicates $\operatorname{det} \tilde{I}_{\theta}(\theta)=-1$, therefore

$$
\operatorname{det} \tilde{H}_{\theta}(\theta)=-\operatorname{det} H_{\theta}(\theta)=\left|\lambda_{1}(\theta)\right| \prod_{j=d+2}^{n} \lambda_{j}(\theta)=|\operatorname{det} H(\theta)|,
$$

where $\lambda_{j}(\theta)$ are the eigenvalues of $H(\theta)$ :

$$
\lambda_{1}(\theta)<0=\lambda_{2}(\theta)=\cdots=\lambda_{d+1}(\theta)<\lambda_{d+2}(\theta) \leq \cdots \leq \lambda_{n}(\theta),
$$

and $\operatorname{det} H(\theta)$ is the product of all the nonzero eigenvalues of $H(\theta)$. (A.28) can be expressed by $H(\theta)$ :

$$
\begin{aligned}
& \frac{C_{0}}{\pi} e^{-\frac{V_{s}}{\epsilon}} \int_{[0,1]^{d}} J(\theta)(2 \pi \epsilon)^{\frac{n-d}{2}} \frac{|\mu(\theta)|}{|\tilde{\operatorname{eet} H}(\theta)|^{\frac{1}{2}}} d \theta \\
= & \frac{C_{0}}{\pi} e^{-\frac{V_{s}}{\epsilon}} \int_{[0,1]^{d}} J(\theta)(2 \pi \epsilon)^{\frac{n-d}{2}} \frac{|\mu(\theta)|}{\left|\lambda_{1}(\theta)\right|^{\frac{1}{2}} \prod_{j=d+2}^{n}\left(\lambda_{j}(\theta)\right)^{\frac{1}{2}}} d \theta .
\end{aligned}
$$

When $d=1$ and the eigenvalues are independent of $\theta$, this becomes

$$
\frac{C_{0}}{\pi} e^{-\frac{V_{s}}{\epsilon}}(2 \pi \epsilon)^{(n-1) / 2} \frac{|\mu|}{\left|\lambda_{1}\right|^{\frac{1}{2}} \prod_{j=3}^{n}\left(\lambda_{j}\right)^{1 / 2}} \int_{0}^{1}\left\|\gamma^{\prime}(\theta)\right\|_{2} d \theta .
$$

For the RHS of (A.18), Laplace's method is still valid. If the eigenvalues of the Hessian matrix $\mathrm{H}$ at $a$ are

$$
0<\lambda_{1}^{a} \leq \lambda_{2}^{a} \leq \cdots \leq \lambda_{n}^{a}
$$

then we obtain the nucleation rate

$$
\begin{aligned}
k & =\frac{|\mu|}{2 \pi}(2 \pi \epsilon)^{-\frac{1}{2}} \sqrt{\frac{\operatorname{det} H(a)}{|\operatorname{det} H(c)|}} \int_{0}^{1}\left\|\gamma^{\prime}(\theta)\right\|_{2} d \theta e^{-\frac{\Delta V}{\epsilon}} \\
& =\frac{|\mu|}{2 \pi}(2 \pi \epsilon)^{-\frac{1}{2}} \frac{\prod_{j=1}^{n}\left(\lambda_{j}^{a}\right)^{1 / 2}}{\left|\lambda_{1}\right|^{\frac{1}{2}} \prod_{j=3}^{n}\left(\lambda_{j}\right)^{1 / 2}} \int_{0}^{1}\left\|\gamma^{\prime}(\theta)\right\|_{2} d \theta e^{-\frac{\Delta V}{\epsilon}} .
\end{aligned}
$$

A.3. The case when $A$ is positive semidefinite. We generalize the results in A.1 and A.2 to the symmetric positive semidefinite case in this subsection. Suppose $A$ can be diagonalized as $U^{T} A U=\operatorname{diag}\left\{\lambda_{1}, \lambda_{2}, \cdots, \lambda_{l}, 0, \cdots, 0\right\}$, where $\lambda_{i}>0, i=$ $1,2, \cdots, l, U^{T} U=I$. That is, we assume $A$ has eigenvalue 0 with multiplicity $n-l$. Decomposing $U=\left(U_{1}, U_{2}\right), U_{1} \in M_{n, l}, U_{2} \in M_{n, n-l}$, then each column of $U_{2}$ is an eigenvector of $A$ corresponding to eigenvalue 0 . We also have $U_{2}^{T} \sigma=0$. In this case, letting $Y_{t}=U^{T} X_{t},\left(\right.$ A.1) can be written as a nSDE for variable $Y_{t}$ :

$$
d Y_{t}=-U^{T} A U \nabla_{y} V\left(Y_{t}\right) d t+\sqrt{2 \epsilon} U^{T} \sigma d W_{t} .
$$

From the above assumption, it is easy to see that only the first $l$ components of $Y_{t}$ are nontrivial and therefore we introduce the variable $Z_{t}=P Y_{t}$, where $P=$ $\left(I_{l \times l}, 0_{l \times(n-l)}\right) \in M_{l, n}, Z_{t} \in \mathbb{R}^{l}$, to reduce (A.32) to

$$
d Z_{t}=-P U^{T} A U P^{T} \nabla_{z} V\left(Z_{t}\right) d t+\sqrt{2 \epsilon} P U^{T} \sigma d W_{t}
$$


Letting $\tilde{A}=P U^{T} A U P^{T}, \tilde{\sigma}=P U^{T} \sigma$, then

$$
d Z_{t}=-\tilde{A} \nabla_{z} V\left(Z_{t}\right) d t+\sqrt{2 \epsilon} \tilde{\sigma} d W_{t} .
$$

What is more, $\tilde{A}=\tilde{\sigma} \tilde{\sigma}^{T}$ still holds and $\tilde{A}=\operatorname{diag}\left\{\lambda_{1}, \lambda_{2}, \cdots, \lambda_{l}\right\}$ is positive definite, and thus the derivation of the nucleation rate formula in A.1 and A.2 is valid for $Z_{t}$. Notice that the dynamics of (A.1) for $X_{t}$ and (A.34) for $Z_{t}$ are equivalent. The trajectory of $X_{t}$ spans a $l$-dimensional subspace $\mathbb{X}_{l}$ in the whole space $\mathbb{R}^{n}$ and the basin of attraction $\Omega_{x} \in \mathbb{X}_{l}$ obviously. Here we derive the nucleation rate formula for $X_{t}$ by changing the variables back.

When the potential (A.34) has a unique saddle point in $\partial \Omega_{z},(A .18)$ holds for $Z_{t}$ :

$$
\epsilon^{\frac{1}{2}} \int_{\partial \Omega_{z}} e^{-\frac{V(z)}{\epsilon}}\left(C_{0}+C_{2}\left(z^{\prime}\right)\right) \sqrt{\frac{2 k\left(z^{\prime}\right)}{\pi}} d \sigma=e^{-\frac{K}{\epsilon}} \int_{\Omega_{z}} e^{-\frac{V(z)}{\epsilon}} d z
$$

Since the change of variable can be viewed as rotation followed by projection, the inner product in $k\left(z^{\prime}\right)$ is invariant and we can change the variable from $z$ to $x$ :

$$
\epsilon^{\frac{1}{2}} \int_{\partial \Omega_{x}} e^{-\frac{V(x)}{\epsilon}}\left(C_{0}+C_{2}\left(y^{\prime}\right)\right) \sqrt{\frac{2 k\left(y^{\prime}\right)}{\pi}} d \sigma=e^{-\frac{K}{\epsilon}} \int_{\Omega_{x}} e^{-\frac{V(x)}{\epsilon}} d x
$$

and the nucleation rate formula can be obtained in the same way:

$$
k=\frac{|\mu|}{2 \pi} \sqrt{\frac{\operatorname{det} H^{\dagger}(a)}{\left|\operatorname{det} H^{\dagger}(c)\right|}} e^{-\frac{\Delta V}{\epsilon}},
$$

where $H^{\dagger}(a)$ and $H^{\dagger}(c)$ are the Hessian matrix confined to $\mathbb{X}_{l}$ at $a$ and $c$ respectively.

When the potential is degenerate, following a similar approach to that in A.2 and changing the variables, we have

$$
\begin{aligned}
& \epsilon^{\frac{1}{2}} \int_{\partial \Omega_{z}} e^{-\frac{V(z)}{\epsilon}}\left(C_{0}+C_{2}\left(z^{\prime}\right)\right) \sqrt{\frac{2 k\left(z^{\prime}\right)}{\pi}} d \sigma \\
= & \frac{C_{0}}{\pi} e^{-\frac{V_{s}}{\epsilon}} \int_{[0,1]^{d}} J(\theta)(2 \pi \epsilon)^{(l-d) / 2} \frac{|\mu(\theta)|}{\left|\operatorname{det} H_{\theta}^{\dagger}(\theta)\right|^{\frac{1}{2}}} d \theta,
\end{aligned}
$$

where $H_{\theta}^{\dagger}(\theta)$ is the Hessian matrix confined to $\mathbb{X}_{l}$ and $M(\theta)^{\perp}$. When $d=1$ and the eigenvalues are independent of $\theta$, we obtain the nucleation rate

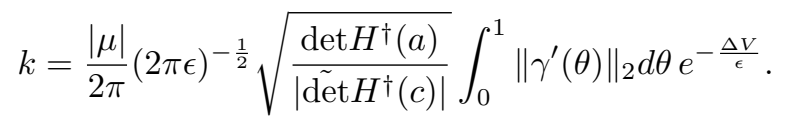

\section{REFERENCES}

[1] P. Hänggi, P. Talkner, and M. Borkovec, Reaction-rate theory: Fifty years after Kramers, Rev. Mod. Phys., 62, 251-341, 1990.

[2] E. Pollak and P. Talkner, Reaction rate theory: What it was, where is it today and where is it going? Chaos, 15, 026116, 2005.

[3] W. E and E. Vanden-Eijnden, Transition-path theory and path-finding algorithms for the study of rare events, Annu. Rev. Phys. Chem., 61, 391, 2010.

[4] M.I. Freidlin and A.D. Wentzell, Random Perturbations of Dynamical Systems, SpringerVerlag, New York, Second (ed.), 1998. 
[5] W. E, W. Ren, and E. Vanden-Eijnden, Minimum action method for the study of rare events, Commun. Pure Appl. Math., 52, 637-656, 2004.

[6] E. Vanden-Eijnden and M. Heymann, The geometric minimum action method for computing minimum energy paths, J. Chem. Phys., 128, 061103, 2008.

[7] W. E, W. Ren, and E. Vanden-Eijnden, String method for the study of rare events, Phys. Rev. B, 66, 052301, 2002.

[8] G. Henkelman and H. Jónsson, Improved tangent estimate in the nudged elastic band method for finding minimum energy paths and saddle points, J. Chem. Phys., 113, 9978, 2000.

[9] G. Henkelman and H. Jónsson, A dimer method for finding saddle points on high dimensional potential surfaces using only first derivatives, J. Chem. Phys., 111, 7010-7022, 1999.

[10] W. E and X. Zhou, The gentlest ascent dynamics, Nonlin., 24, 1831, 2011.

[11] B.J. Matkowsky and Z. Schuss, The exit problem for randomly perturbed dynamical systems, SIAM J. Appl. Math., 33, 365-382, 1977.

[12] W. E and E. Vanden-Eijnden, Towards a theory of transition paths, J. Stat. Phys., 123, 503-523, 2006.

[13] P.C. Hohenberg and B.I. Halperin, Theory of dynamic critical phenomena, Rev. Mod. Phys., 49, 435-479, 1977.

[14] G. Da Prato and A. Debussche, Stochastic Cahn-Hilliard equation, Nonlin. Anal., 26, 241-263, 1996.

[15] P.W. Bates and P.C. Fife, The dynamics of nucleation for the Cahn-Hilliard equation, SIAM J. Appl. Math., 53, 990-1008, 1993.

[16] D. Blömker, B. Gawron, and T. Wanner, Nucleation in the one-dimensional stochastic CahnHilliard model, Discrete Cont. Dyn. Sys. A, 27, 25-52, 2010.

[17] J. Feng, Large deviation for stochastic Cahn-Hilliard equation, Meth. Funct. Anal. Topol., 9, 333-356, 2003.

[18] A. Debussche and L. Zambotti, Conservative stochastic Cahn-Hilliard equation with reflection, Ann. Prob., 35, 1706-1739, 2007.

[19] M. Grinfeld and A. Novick-Cohen, Counting stationary solutions of the Cahn-Hilliard equation by transversality arguments, Proc. Roy. Soc. Edinburgh Sect. A, 125, 351-370, 1995.

[20] E. Vanden-Eijnden and M.G. Westdickenberg, Rare events in stochastic partial differential equations on large spatial domains, J. Stat. Phys., 131, 1023-1038, 2008.

[21] T.W. Heo, L. Zhang, Q. Du, and L.Q. Chen, Incorporating diffuse-interface nuclei in phasefield simulations, Scripta Mater., 63, 8-11, 2010.

[22] W. Ren, Numerical Methods for the Study of Energy Landscapes and Rare Events, PhD thesis, Princeton University, 2002.

[23] W. E, W. Ren, and E. Vanden-Eijnden, Simplified and improved string method for computing the minimum energy paths in barrier-crossing events, J. Chem. Phys., 126, 164103, 2007.

[24] T. Qian, W. Ren, and P. Sheng, Current dissipation in thin superconducting wires: A numerical evaluation using the string method, Phys. Rev. B, 72, 014512, 2005.

[25] C. Jin, W. Ren, and Y. Xiang, Computing transition rates of thermally activated events in dislocation dynamics, Scripta Mater., 62, 206-209, 2010.

[26] W. E, W. Ren, and E. Vanden-Eijnden, unpublished manuscript.

[27] G.O. Roberts and J.S. Rosenthal, Optimal scaling for various metropolis-hastings algorithms, Stat. Sci., 16, 351-367, 2001.

[28] G. Ariel and E. Vanden-Eijnden, Testing transition state theory on Kac-Zwanzig model, J. Stat. Phys., 126, 43-73, 2007.

[29] L. Lin, X. Cheng, W. E, A. Shi, and P. Zhang, A numerical method for the study of nucleation of ordered phases, J. Comp. Phys., 229, 1797-1809, 2010.

[30] R. Barry and R.K. Pace, Monte Carlo estimates of the log determinant of large sparse matrices, Lin. Alg. Appl., 289, 41-54, 1999.

[31] R.K. Pace and J.P. LeSage, Chebyshev approximation of log-determinants of spatial weight matrices, Comp. Stat. Data Anal., 45, 179-196, 2004.

[32] R. Forman, Functional determinants and geometry, Invent. Math., 88, 447-493, 1987.

[33] G.V. Dunne, Functional determinants in quantum field theory, J. Phys. A: Math. Theor., 41, 304006, 2008

[34] E. Vanden-Eijnden, Computer simulations in condensed matter systems: From materials to chemical biology: Volume 1, M. Ferrario, G. Ciccotti, and K. Binder, (eds), Lecture Notes in Physics, Springer-Verlag, 703, 453-493, 2006. 